\title{
Electron tomography of cells
}

\author{
Lu Gan' and Grant J. Jensen',2*
}

' Division of Biology, California Institute of Technology, Pasadena, CA 91125 , USA

${ }^{2}$ Howard Hughes Medical Institute, California Institute of Technology, Pasadena, CA 91 I25, USA

\begin{abstract}
The electron microscope has contributed deep insights into biological structure since its invention nearly 80 years ago. Advances in instrumentation and methodology in recent decades have now enabled electron tomography to become the highest resolution threedimensional (3D) imaging technique available for unique objects such as cells. Cells can be imaged either plastic-embedded or frozen-hydrated. Then the series of projection images are aligned and back-projected to generate a 3D reconstruction or 'tomogram'. Here, we review how electron tomography has begun to reveal the molecular organization of cells and how the existing and upcoming technologies promise even greater insights into structural cell biology.
\end{abstract}

\section{Introduction 28}

\section{Technology and techniques}
2.I. Specimen preparation 29
2.2. Data collection 31
2.3. Reconstruction 33
2.4. Analysis 34

3. Example applications of electron tomography 36
3.1. Membrane systems involved in vesicular traffic 37
3.2. Eukaryotic macromolecular complexes 37
3.3. Tomography of tissues 41
3.4. Bacterial ultrastructure 41
3.5. Virus-cell interactions 45

\section{Future developments 46}
4.I. Better specimens 46
4.2. Better images 47
4.3. Better analyses 47

\section{Conclusion 48}

\section{Acknowledgements}

\section{References 49}

* Author for correspondence: G. J. Jensen. Tel.: (626) 395 - 8827; Fax: (626) 395 - 5730; Email: jensen@caltech.edu 


\section{Introduction}

The science of cell biology has been driven by advances in imaging technologies ever since the discovery of cells by Robert Hooke and Antonie van Leeuwenhoek in the 17th century. As optics improved over the next three centuries, so did our knowledge of cell ultrastructure and function. The development of chromogenic stains enabled the visualization of otherwise 'invisible' intracellular bodies, including chromosomes inside the nucleus. In the late 19th century Ernst Abbe recognized, however, that the resolution of light microscopes would ultimately be limited to about half the wavelength of light.

The invention of the electron microscope by Ruska in the early 1930s and the availability of commercial instruments shortly thereafter promised a new era of cell biology in which resolution could be extended by at least two orders of magnitude. Despite this transformative technological advance, the first images of subcellular structures such as ribosomes, viruses and the cytoskeleton were not obtained for approximately two more decades. Some of the principal challenges were that biological specimens are hydrated and radiation sensitive, exhibit poor intrinsic contrast, and are often too thick to be imaged intact. To solve these problems, methods were developed to chemically fix and stain specimens, embed them in plastic resins, and section them with a microtome. Together, these techniques enabled a 'Golden age' of rapid discovery in the 1950 s to 1960s (Fig. 1).

Electron microscopy (EM) images are 2D projections. To gain the perspective of depth, stereoscopy was introduced, in which two images are taken of a specimen - one at positive tilt and the other at negative tilt. The pair of images were viewed with a stereoscope, and quantitative data could be generated by measuring relative displacements of objects perpendicular to the tilt axis and then calculating their separation in ' $Z$ ' (the direction parallel to the electron beam) using the known tilt angles. But since stereoscopy uses only two views of the specimen, the resolution in $Z$ is limited.

Another way to obtain 3D information is to cut a sample into a series of thin sections and image each section individually. As each section represents a resolution element in $Z$, an entire cell can then be reconstructed in $3 \mathrm{D}$ by computationally stacking the images. Reconstructions from serial sections have an anisotropic resolution of $\sim 1-2 \mathrm{~nm}$ in the cutting plane (limited by the stain granularity), but only $\geqslant 20 \mathrm{~nm}$ perpendicular to the cutting direction (limited by how thin sections can be cut and handled) (Mastronarde et al. 2000). Fine structural details of cellular organization are often further obfuscated by the losses that occur on the cutting planes, sample shrinkage and warping caused by radiation damage, and the difficulty of tracking features between sections (especially features that lie in a cutting plane). Nevertheless, since it is relatively straightforward to do in any laboratory with an EM, this method has been used since the 1950s to map out the ultrastructure of various eukaryotic cells and tissues. As an example of the power of this technique, the Caenorbabditis elegans 'connectome' - the wiring diagram of all 302 neurons in this millimeter-long worm - was reconstructed by serial EM (White et al. 1986).

A third method to obtain high-resolution ' $3 \mathrm{D}$ ' EM images of at least the surface of biological objects is scanning electron microscopy. Newer methods that combine sequential focused-ion beam milling with SEM (also called ion-abrasion scanning electron microscopy) are beginning to reveal internal detail as well, as demonstrated in a recent study of murine liver (Murphy et al. 2010). In this 'slice-and-scan' approach, a heavy-metal-stained, plastic-embedded block of cells is imaged on one face by SEM, then milled to expose a fresh surface and imaged again. This 'sequential imaging' technique eliminates the distortion artifacts that may occur when adjacent 


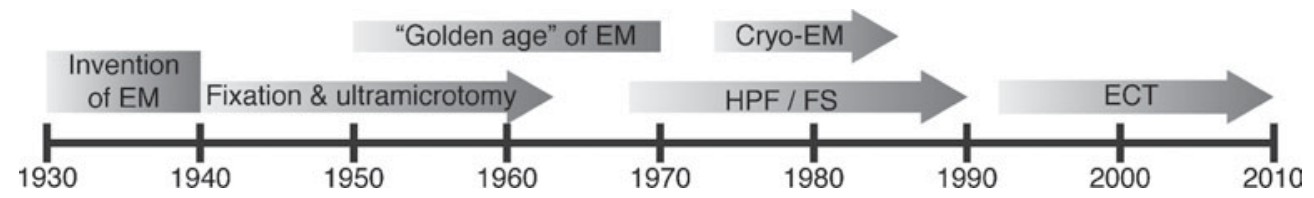

Fig. 1. Timeline of key developments. Key technologies and applications of EM are shown. The gray gradients emphasize that each of these development took many years.

(a)

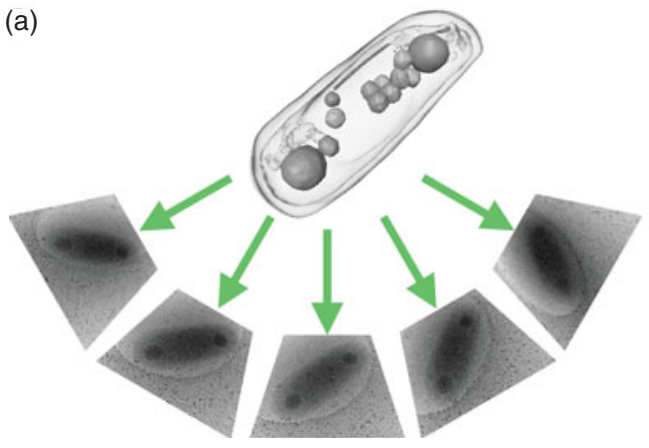

(b)

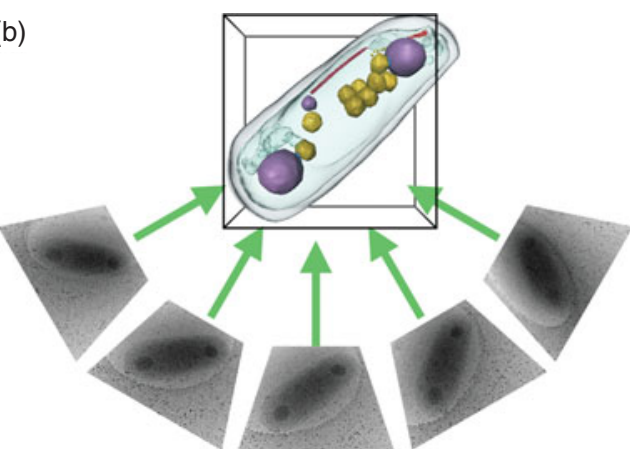

Fig. 2. Principle of electron tomography. (a) A tilt series of projection images is acquired from the object as it is incrementally tilted. (b) The tomogram is generated by back-projecting the aligned projection images.

sections are imaged separately for serial-section reconstruction. It is not yet known, however, how deeply the interior of the block can be uniformly stained, milled and imaged, and how this will affect the range of structures that can be examined.

Alternatively, samples can be imaged to a resolution of $\sim 2-8 \mathrm{~nm}$ in all three directions without losses or distortions between sections through electron tomography (Fig. 2). Specimens are incrementally tilted in a transmission EM through a range up to $\pm 70^{\circ}$ and imaged at each step. Each image in this 'tilt series' represents a different view of the specimen. The images are aligned and then 'back-projected' to generate a 3D reconstruction or 'tomogram', of the specimen. Electron tomography is therefore the highest resolution technique available today to image unique biological specimens such as cells.

In this review, we will summarize the current methods and technologies to prepare a specimen, collect data, generate a tomogram, and finally interpret and analyze the result (for additional technical details, see also Frank, 2006; Koster et al. 1997; Lucic et al. 2005). While electron tomography can be used to image even individual macromolecular complexes (Murphy $\&$ Jensen, 2005), here we limit ourselves to the application of electron tomography to cells. The role of electron tomography in solving important cell biology problems will then be illustrated with a few example studies of eukaryotic and bacterial ultrastructure and virus-cell interactions.

\section{Technology and techniques}

\section{I Specimen preparation}

EM specimens must satisfy two important requirements to produce useful phase-contrast EM images: they must be stable within the microscope's high vacuum and they must be thin. Since cells are composed mostly of water, their molecular structure must be fixed (mechanically 
stabilized) somehow before they can be imaged in the microscope. Samples must be thin because while phase contrast arises from the interference between unscattered and elastically scattered electrons, inelastically scattered electrons emerge from the sample with increased wavelengths and therefore focus differently, blurring the image. Inelastically scattered electrons are therefore typically removed from the image with an energy filter. There is no exact limit on how thin a specimen must be, but because the probability of electrons being inelastically scattered increases with sample thickness, and the path length of the electrons through the sample increases with tilt angle $\alpha$ by $\sim 1 / \cos (\alpha)$, sample thickness is generally limited to about $200 \mathrm{~nm}$ in $100 \mathrm{kV}$ instruments and approximately twice than that in $300 \mathrm{kV}$ instruments. While higher voltage (up to several $\mathrm{MeVs}$ ) EMs exist, these instruments are extremely rare and only moderately expand (approximately another doubling) the permissible specimen thickness.

The first method that was developed to stabilize a biological specimen is chemical fixation. Cells (or small pieces of tissue) can be fixed at room temperature with cross-linking agents like glutaraldehyde. Next, the cells are typically post-fixed with osmium tetroxide, a cross-linking agent that also serves as a heavy-metal stain. The cells are then dehydrated with an organic solvent such as acetone or ethanol and infiltrated with embedding resin. The resin is polymerized, yielding a mechanically stable 'block' of cells which can be subsequently sectioned in a microtome. To enhance the contrast, the cells can be post-stained with a heavy metal salt such as uranyl acetate or lead citrate (usually after microtomy). Because this 'traditional' chemical fixation method can be applied to almost any specimen and the samples can be imaged at room temperature in almost any EM, it has been widely used for decades and has generated a vast archive of cellular images. Unfortunately, because fixation occurs in tens of seconds or longer and different parts of cells are fixed at different rates, dynamic cellular structures often disassemble and molecules that do not normally interact can be cross-linked into un-physiological complexes (McDonald \& Auer, 2006).

Cellular structures can be better preserved if they are quickly frozen. Crystalline ice, however, can damage proteins and can generate image artifacts when the diffraction condition is satisfied (Dubochet et al. 1988). A family of techniques have therefore been developed to rapidly cool cells to a critical temperature $\left(-135^{\circ} \mathrm{C}\right)$ below which water can be immobilized into a crystal-free, amorphous state. Cells up to a few $\mu \mathrm{m}$ thick can be applied to an EM grid, drained of excess fluid, and then plunged into a cryogen such as liquid ethane, which drops the temperature so quickly $(>10000 \mathrm{~K} / \mathrm{s}$ ) that the water molecules do not have time to rearrange into ice crystals before they stop moving (Dubochet et al. 1988). Plunge-freezing suspends molecules in 'vitreous' ice in a life-like, 'frozen-hydrated' state. It is also efficient and inexpensive, so it is the vitrification method of choice when possible. Plunge-freezing only works, however, on samples that are less than $\sim 15 \mu \mathrm{m}$ thick, such as single monolayers of eukaryotic cells. If the specimen is too thick to be plunge-frozen, it can be vitrified by high-pressure-freezing. In this method, the sample is pressurized to $\sim 2000$ bar and then rapidly cooled by liquid nitrogen jets (Moor, 1987). Since the pressure suppresses ice crystal formation, specimens up to $\sim 250 \mu \mathrm{m}$ thick can be frozen vitreously (Gilkey \& Staehelin, 1986).

Frozen-hydrated specimens can either be imaged directly (if they are thin enough, see below), or chemically fixed at low temperature, a process called freeze-substitution (FS). For FS, samples are transferred into a cold $\left(-90^{\circ} \mathrm{C}\right)$ acetone (or methanol) solution containing fixatives such as glutaraldehyde and tannic acid. The specimen is then slowly warmed to approximately $-40{ }^{\circ} \mathrm{C}$ and placed in another acetone solution containing osmium tetroxide and uranyl acetate. The solid water within the cells is gradually replaced by these fixative-containing solvents, which cross-link 
macromolecules while the cells are still well below the melting point of water. Freeze-substituted cells are then embedded in low-temperature-polymerizing plastics and finally sectioned at room temperature by standard microtomy methods. FS results in specimen preservation that is substantially better than what is achieved by traditional room-temperature fixation. Tomography of individual or serial thick ( $\sim 400 \mathrm{~nm}$ ) sections can yield 3D images of substantial portions of even large eukaryotic cells (Marsh, 2007). Such specimens still suffer from some fixation and dehydration artifacts, however, and the stain only approximately represents the structures of the molecules it is bound to.

The development of 'cryo-EM (cryoelectron microscopy)' in the 1970s and 1980s allowed frozen-hydrated samples to be kept frozen while in the microscope and therefore imaged directly without fixation or plastic embedding (Adrian et al. 1984; Dubochet et al. 1983; Taylor \& Glaeser, 1974). Cells can be plunge-frozen as 'whole-mount' specimens and then regions thinner than $\sim 0.5 \mu \mathrm{m}$ can be imaged directly. Suitable specimens include many bacterial and archael cells, some picoeukaryotic cells, and the thin margins of higher eukaryotes. Plunge-frozen cells are free from the artifacts associated with dehydration, chemical-fixation, plastic-embedding and heavymetal staining, but surface tension in the thin film produced before plunge-freezing can flatten cells, and evaporation (if not controlled) can change osmotic pressures (Frederik \& Hubert, 2005). The vast majority of eukaryotic cells, however, are much thicker than $1 \mu \mathrm{m}$, so they must still be sectioned after plunge-freezing or high-pressure freezing). One way to do this is with a cryomicrotome, which is kept below the vitrification temperature. Cryosections as thin as $40 \mathrm{~nm}$ can be cut, transferred onto an EM grid, and imaged in a cryo-EM. While cryosectioning causes knife marks and crevasses near the surfaces (Dubochet et al. 2007), the center can still be well preserved, except for some compression. Thus, any cell can now be imaged in a near-native, life-like state in 3D to 'molecular' (2-5 nm) resolution (Milne \& Subramaniam, 2009; Tocheva et al. 2010).

The quality of a tomogram, whether it is of a cryo- or room-temperature sample, is limited by the accuracy to which the tilt series of images can be aligned. While tilt series can sometimes be aligned by cross-correlation (so-called 'fiducial-less' alignment) (Winkler \& Taylor, 2006), gold beads are typically added to the specimen during preparation to serve as fiducial markers that can be tracked from one image to the next. For plastic sections, gold fiducials are typically applied to the top and bottom of the sample after microtomy, sectioning and staining. For plunge-frozen samples, gold fiducials are simply added to the liquid sample and mixed with the cells before freezing. Mixing gold fiducials with the cells before freezing also works well for cryosections of small cells (cells that fit within single images), since a good number of fiducials are then present around the periphery of each image. The deposition of fiducials onto cryosections of larger cells is more challenging, however, because they must be added at cryogenic temperatures to the surfaces of sections. For this, quantum dots or gold nanoparticles can be dissolved in isopentanebased cryogenic solvents (Gruska et al. 2008; Masich et al. 2006). Depending on the nature of the experiment, other technical factors must also be considered, including the type of grid, glowdischarging conditions, thickness and design of carbon film, etc. (Iancu et al. 2006a; McDonald, 2009).

\subsection{Data collection}

Plastic-embedded and frozen-hydrated samples react very differently to electron-beam exposure. Plastic-embedded samples first lose mass rapidly and shrink, but then stabilize (Bennett, 
1974). Plastic sections are therefore first 'pre-irradiated', and then imaged without excessive concern for subsequent dose. In contrast, frozen-hydrated cells are highly radiation sensitive, with damage manifest as a progressive loss of high resolution detail followed in many cases by the appearance of 'bubbles' that catastrophically distort the cell (Iancu et al. 2006b). The dose tolerance of a new frozen-hydrated sample can be explored by iteratively imaging a test region and noting the onset of fine detail loss and the appearance of bubbles. In practice this is usually followed by recording full tilt series at different total doses and comparing the resulting reconstructions (Briegel et al. 2006). Total applied doses between 60 and 200 electrons $/ \AA^{2}$ are typical. The electron dose used to find the target, focus and establish conditions for image acquisition must therefore be minimized. The electron beam is set to a diameter just slightly larger than the target field of view, and it is electronically shuttered when images are not being acquired. All of the focusing, stage height, and re-centering adjustments are done on a sacrificial part of the grid near the target.

In a typical cryotomographic data collection session, after a grid is inserted, it is brought to approximate eucentric height and a low-magnification, low-dose, montage 'atlas' is collected of its central region. Targets are then marked on the atlas through careful inspection on the computer screen while the beam is blanked (Suloway et al. 2009). Once the target list is prepared, the rest of the process is automated: the microscope moves the stage to center the first target under the beam; verifies correct positioning with additional low-magnification, low-dose images; deflects the beam off the target to a sacrificial 'focus' position; refines eucentric height; resets focus; centers the energy slit; redirects the (blanked) beam back onto the target; records the first image; tilts the sample one increment; records the second image; tilts the sample another increment; measures the displacement of the target between the first two images on the camera through cross-correlation; models the movement of the sample in 3D (Zheng et al. 2004); applies beam shifts above and below the sample to keep the target in the center of the image; adjusts the objective lens current to maintain constant focus; records the next image; etc. The beam can also be deflected periodically off the specimen to confirm focusing as needed.

Obviously, the process of shifting and refocusing the beam, managing the dose applied, and recording the images for even one tilt series is tedious! Several software packages are now available which acquire grid atlases, facilitate target selection, and then automatically collect tilt series, including SerialEM (Mastronarde, 2005), Leginon (Suloway et al. 2009), the TOM ${ }^{2}$ Toolbox (soon to be released), UCSF Tomo (Zheng et al. 2009) and Xplore3D (FEI Inc.). With the assistance of these packages, up to several tens of tilt series can now be collected automatically in a single (24-hour) day.

The tilt increment sets a limit on the theoretical resolution of the reconstruction. The Crowther criterion (Crowther et al. 1970) relates the number of views $m$ to the thickness of the object $D$ and the desired resolution $d$ :

$m \approx \pi D / d$

Therefore, to achieve $\sim 10 \mathrm{~nm}$ resolution in a tomogram of a 500-nm-thick bacterial cell, 157 images $\left(0.87^{\circ}\right.$ tilt increment) must be acquired over the full $180^{\circ}$ of tilt range. For tomography of bacteria, this increment is usually rounded to $1^{\circ}$. Theoretically, some advantage can be gained by varying the tilt increment as a function of tilt angle (Lee et al. 2008; Saxton et al. 1984), but it is not commonly done.

Since good images of samples tilted beyond $\sim 60-70^{\circ}$ cannot usually be obtained (both because the effective path length through the specimen becomes too thick and because the 
specimen holder sometimes blocks the beam), there is a wedge-shaped region of data in reciprocal space that is missed, decreasing the resolution of the reconstruction parallel to the beam. To minimize this 'missing-wedge' artifact, an additional tilt series can be acquired about an axis orthogonal to the first, resulting in a 'dual-axis' tomogram that has a less-severe 'missing pyramid' artifact, and therefore more isotropic resolution (Mastronarde, 1997). While dual-axis tomography is commonly used for plastic sections, it is still unusual for frozen-hydrated samples, mostly because it requires that the dose be split between two tilt series, trading gains in reciprocal space coverage for losses in individual image clarity and significant extra effort (Iancu et al. 2005; McEwen et al. 1995).

Plastic sections generate substantial amplitude contrast due to the presence of heavy-metal stains and can therefore be imaged close to focus. Frozen-hydrated specimens, however, generate very little amplitude contrast and must be imaged using a large defocus to generate phase contrast. Oscillations of the contrast transfer function must not corrupt details of interest, however (see below), so defocus settings are chosen to be as high as possible without limiting the resolution more than what is imposed by radiation damage. Likewise, magnifications are chosen to be as low as possible to yield larger fields of view, but without further limiting resolution (the pixel size must be 2-3 times smaller than the target resolution). In practice, magnifications corresponding to pixel sizes on the camera of $\sim 1 \mathrm{~nm}$ and defoci of $4-12 \mu \mathrm{m}$ (at $300 \mathrm{kV}$ ) are typical.

\subsection{Reconstruction}

The mathematics for reconstructing a tomogram in real space was formulated by Radon in the early 1900s. A Radon transform of a 3D volume is a complete set of 2D projections through the object around some axis. This is applicable to electron tomography because TEM images are projections. Given a complete set of projections, the original 3D object can be reconstructed by a process called 'back-projection', in which the individual 2D images are 'smeared' through a common space where their densities are summed (Penczek, 2010a). A tomogram can also be reconstructed using mathematically equivalent methods in reciprocal space. According to the projection theorem, the Fourier transform of a $2 \mathrm{D}$ projection image is also a central section through the object's 3D Fourier transform perpendicular to the direction of projection. The 3D Fourier transform can be reconstituted from multiple 2D transforms of images taken from different orientations, and then Fourier-inverted to produce a 3D real-space image. Iterative algorithms can also be used, either alone or to refine initial results (Gilbert, 1972; Herman et al. 1973).

In practice, tilt series of images must first be aligned (translated, rotated and stretched) to compensate for slight differences in magnification, image rotation and translation. The precise tilt angle and tilt axis of each image must also be determined before 3D reconstruction. There are now many software packages available for these tasks including IMOD (Mastronarde, 1997), the TOM Toolbox (Nickell et al. 2005), SPIDER (Shaikh et al. 2008), EM3D (Ress et al. 1999), TxBR (Lawrence et al. 2006), protomo (Winkler, 2007), TomoJ (Messaoudii et al. 2007) and Bsoft (Heymann et al. 2008). Another program, RAPTOR, can automatically find and track fiducial markers, eliminating one of the bottlenecks in high-throughput electron tomography by automating the entire reconstruction process (Amat et al. 2008).

The resolution of a cryo-EM image is limited by the electron microscope's contrast transfer function $(\mathrm{CTF})$, which undergoes phase reversals in the low-nanometer resolution range when 
large defoci are used. CTF correction can be done for individual 2D images by fitting power spectra to theoretical models (Penczek, 2010b). CTF correction has enabled near-atomic resolution reconstructions of both 2D crystals (Henderson et al. 1990) and various 'single' particles (Bottcher et al. 1997; Conway et al. 1997), but it is challenging in electron tomography because the images are so low contrast and exhibit a defocus gradient. Nevertheless, CTF-correction programs have now been implemented for electron cryotomography and are showing promising initial results (Fernandez et al. 2006; Winkler, 2007; Xiong et al. 2009; Zanetti et al. 2009). Advances in phase-plate technology and spherical aberration correctors may ultimately make CTF-correction unnecessary (Section 5).

\subsection{Analysis}

The information we seek through cellular tomography is the locations and structures of subcellular components as large as nuclei or as small as individual macromolecules. As a first step, the boundaries of major compartments such as organelles, vesicles and protein microcompartments (Iancu et al. 2010) are typically located. While there have been efforts to automate such feature/edge detection (Noske et al. 2008; Sandberg \& Brega, 2007), the most common way to mark such boundaries is still hand-segmentation (also called modeling). To do this, tomograms can be visualized in either 2D or 3D. Individual 2D slices with arbitrary thickness and orientation are displayed with tools like $3 \mathrm{dmod}$ and Slicer from the IMOD software package (Kremer et al. 1996). Segmented features can then be rendered as 3D surfaces and displayed in any orientation and relative to any other segmented objects. Surface areas, volumes and spatial relationships can be measured (for example, see Marsh et al. 2001).

To facilitate segmentation, contrast can be improved by 'denoising', which consists of algorithms for low-pass filtering, non-linear anisotropic diffusion (Frangakis \& Hegerl, 2001), iterative median filtering (van der Heide et al. 2007), or non-local means filtering (Wei \& Yin, 2010). With the exception of the low-pass filter, denoising algorithms are highly parameterdependent, and must be used with caution to prevent the generation of artifacts.

Like compartment boundaries, individual large macromolecular structures can also be identified and inspected. The largest and most distinct can be identified by eye, including for instance microtubules, branched actin networks, basket-shaped nuclear pore complexes (NPCs), triskelion-shaped clathrin coats and even ribosomes. This is usually done by scanning up and down through 2D slices, as this resolves the superposed 3D complexity of crowded cells. Alternatively, isosurfaces of 3D densities within subvolumes can be shown using programs such as UCSF Chimera (Pettersen et al. 2004), Amira (Mercury Computer Systems Inc.), or IMOD. Segmentation tools, including watersheds (Volkmann, 2002) and 'magic wands' that find all voxels connected to a starting point with densities above some threshold, can be used to objectively delineate substructures.

Most tomograms contain novel features that would be interesting to identify, and there is usually a long list of known structures that would be interesting to find within the tomogram. If the cell of interest is genetically tractable, specific structures can sometimes be identified by perturbing the abundance of candidate components. Filaments at the predivisional constriction site of Caulobacter crescentus cells, for instance, were confirmed to be FtsZ by overexpressing wildtype and mutant forms of the protein, which altered the abundance and length of the filaments as expected ( $\mathrm{Li}$ et al. 2007). The disadvantage of this approach is that it can require tens of tomograms of multiple strains to establish reliable statistics. 

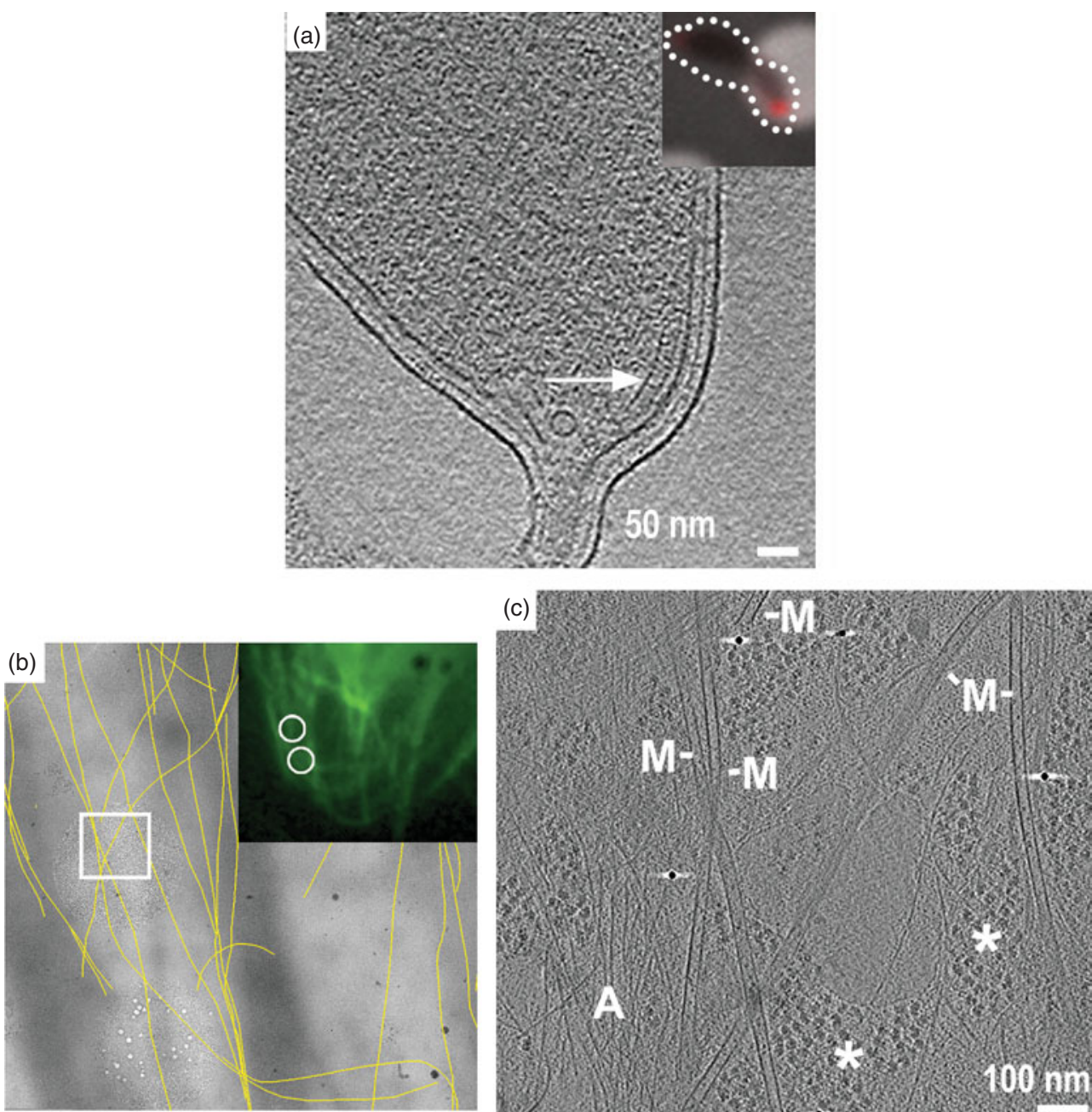

Fig. 3. Correlated light and electron microscopy. (a) Correlation of mCherry-tagged McpA (inset, red) in a light micrograph of a $C$. crescentus cell (inset, dotted outline) and of the same cell in an electron cryotomogram. The chemoreceptor array is indicated by an arrow. (b) Low-magnification cryo-EM image showing the positions of individual segmented microtubules (yellow lines), which match those identified by the $\alpha$-tubulin-GFP fluorescence cryo-light microscopy image of the same cell (upper right panel). (c) Cryotomographic slice of the region boxed in (b) showing microtubules $(\mathrm{M})$, actin filaments (A) and putative ribosomes (asterisks) in a $\mathrm{PtK}_{2}$ cell. Reproduced with permissions from (a) Briegel et al. (2008) and (b, c) (Schwartz et al. 2007).

Structures can also be identified through correlated light and electron microscopy (correlated $\mathrm{LM} / \mathrm{EM}$ ). In one strategy, cells with fluorescently tagged targets are applied to an EM grid, imaged by fluorescence light microscopy at room temperature, plunge-frozen, and then imaged again in the electron microscope (Fig. 3 a). The location in the tomogram of the tagged target is then found by superposing the LM image on the EM image through appropriate transformations. Of course the LM image is of much lower resolution, so the fluorescent focus spreads beyond the target across larger portions of the cell, but the target lies at the center and repeating the experiment can make the correlation unambiguous. This strategy works well when the 
molecular target is stationary within a cell, and has been used for instance to localize the bacterial chemoreceptor cluster at the flagellar pole of C. crescentus cells (Briegel et al. 2008). Alternatively, the recent development of cryo-LM stages (Fig. $3 b, c$ ) now allows recording of fluorescence images of samples that are already plunge-frozen (Chen et al. 2010). This ensures that no molecular movements occur between imaging modalities. Genetically encoded fluorescent tags are better than antibody-targeted tags in all these applications because 'immunofluorescence' requires detergent permeabilization (plus lysozyme-treatment for bacteria), which results in grossly altered cell morphology.

In organisms that are not yet genetically tractable, proteins can be partially localized in 3D by combining immuno-EM of Tokuyasu cryosections with tomography (Ladinsky \& Howell, 2007; Tokuyasu, 1986). While the antibody-gold complex usually only labels the surface of a section, it can provide 3D information if the labeled structure (like a Golgi cisterna; Ladinsky \& Howell, 2007) can be tracked deeper into the tomogram.

Where atomic or high-resolution models are available, cryotomograms can be searched for densities of similar size and shape, a procedure that has been named 'template matching' (Beck et al. 2009; Forster, 2005; Ortiz et al. 2006; Seybert et al. 2006). Ribosomes are particularly recognizable because of their large size $(25 \mathrm{~nm})$ and higher-than-average density (from the phosphorus in ribosomal RNA). While potentially very powerful, extension of this type of correlation analysis ('visual proteomics'; Nickell et al. 2006) to smaller complexes will depend on improvements in image quality and/or the development of better computational searches.

Once a protein or complex is localized and inspected by eye or segmentation, additional information can often be obtained through averaging. Subtomograms that contain the complex of interest are extracted, aligned and averaged, potentially resulting in the elucidation of even high-resolution details. Because the precision of alignment depends on the size of the macromolecular complex and the signal-to-noise ratio of the original tomogram, the most successful applications of this technique to date have been on large dense structures such as the bacterial flagellar motor (Liu et al. 2009; Murphy et al. 2006) and ribosomes (Brandt et al. 2010; Ortiz et al. 2010). Periodic and semi-periodic structures are also amenable to subtomographic averaging analysis because the symmetries present can be detected in the power spectra of appropriately oriented slices. In some cases the signal-to-noise ratio can be increased sufficiently to suggest quasi-atomic models (Al-Amoudi et al. 2007; Briegel et al. 2008).

\section{Example applications of electron tomography}

The first published electron tomography study was of the yeast fatty acid synthase (Hoppe et al. 1974). While the structural insights gained from the resulting tomogram were limited by the chemical fixation and instrumentation, this study was a concrete demonstration that electron tomography of biological specimens was feasible. It took another decade before electron tomography was applied to cellular structures such as Balbiani ring ribonucleoprotein particles (Olins et al. 1983; Skoglund et al. 1986), cilia (McEwen et al. 1986) and chromatin (Belmont et al. 1987). Since then, electron tomography has revealed a wealth of information about cellular ultrastructure, but it is clearly just a beginning. Here we will survey several examples that are by no means comprehensive, but illustrate the kinds and range of questions that can be addressed by tomography. 


\section{I Membrane systems involved in vesicular traffic}

Electron tomography has made - and continues to make - important contributions to our understanding of pleomorphic cellular bodies. Studies of vesicular trafficking are good examples because many vesicles are thinner than the serial thin-sections used for serial-EM reconstructions, and so cannot be well resolved by any other means. An early study of frog saccular hair cells, for example, showed how two classes of vesicles (synaptic and non-synaptic) crowded around the synaptic body in the presynaptic cytoplasm (Lenzi et al. 1999). Electron tomography revealed rare $\Omega$-shaped profiles in the presynaptic membrane, supporting the idea that membrane recycling was occurring and contributing to the enrichment of vesicles in the synaptic cytoplasm (Fig. 4a). In further experiments in which the cells were subjected to prolonged stimulation, the synaptic body-associated vesicles were depleted on the hemisphere closest to the presynaptic membrane (Lenzi et al. 2002). This suggested that when a neuron is stimulated, the synaptic vesicles move along the surface of synaptic bodies toward the presynaptic membrane. These studies are also notable as demonstrations of 'telemicroscopy' technology that was pioneered at University of California, San Diego (Fan et al. 1993).

As another example, the Golgi body (also called Golgi apparatus) is depicted in textbooks as a stack of large flattened vesicles called cisternae. In 3D, the Golgi body is a much more complicated network of fenestrated cisternae, tubules, and both coated and uncoated vesicles. Molecular traffic between the cis- and trans-cisternae is bi-directional and must be highly regulated to ensure proper maturation of lipids and proteins. The first 3D models of this remarkable vesicular switchboard were painstakingly constructed using stereoscopic methods (some examples are in Clermont et al. 1995), but the visualization of details about the connectivity of cisternal tubules and the distribution of different classes of vesicles, for example, required electron tomography. The Golgi body is so big, however, that a single tomogram cannot capture much of its organization. Instead, 'montage' tomograms were acquired from serial sections (Soto et al. 1994) and then merged both laterally and 'vertically' in silico to reconstruct a $4 \mu \mathrm{m}^{3}$ cellular volume (Ladinsky et al. 1999). 3D models of this merged serial tomogram illustrate the complexity of the true organization of the Golgi body (Fig. 4b,c) and also led to the discovery of vesicle-filled 'wells', which are formed by the aligned fenestrae from a series of cisternae. Subsequent studies showed that in secretory cells, cisterna were often connected by tubules (Marsh et al. 2004; Trucco et al. 2004), supporting the idea that the Golgi enzyme retrograde transport mechanism involves more than just vesicular traffic. Ambitious large-scale serial electron-tomography programs are now modeling the Golgi body in medically important systems such as the pancreatic beta cells (Noske et al. 2008).

\subsection{Eukaryotic macromolecular complexes}

Chromosome segregation is carried out by the conserved machines of the mitotic spindle. Spindles capture sister chromatids and mechanically draw them apart. Historic serial-EM studies classified and counted the microtubules in some key model systems (Ding et al. 1993; McIntosh et al. 1985; Winey et al. 1995), but did not reveal much about the ends of the microtubules. Detailed in vitro characterization of the dynamic plus end (Chretien et al. 1995; Mandelkow et al. 1991) and the relatively static minus end (Moritz et al. 2000) showed that the structure of microtubule ends might provide clues about their assembly state and function in vivo. Indeed, tomograms of the Saccharomyces cerevisiae spindle pole body revealed that the microtubule minus ends typically have a tapered cap (Fig. $5 a$ ), supporting the idea that the gamma-tubulin complex 

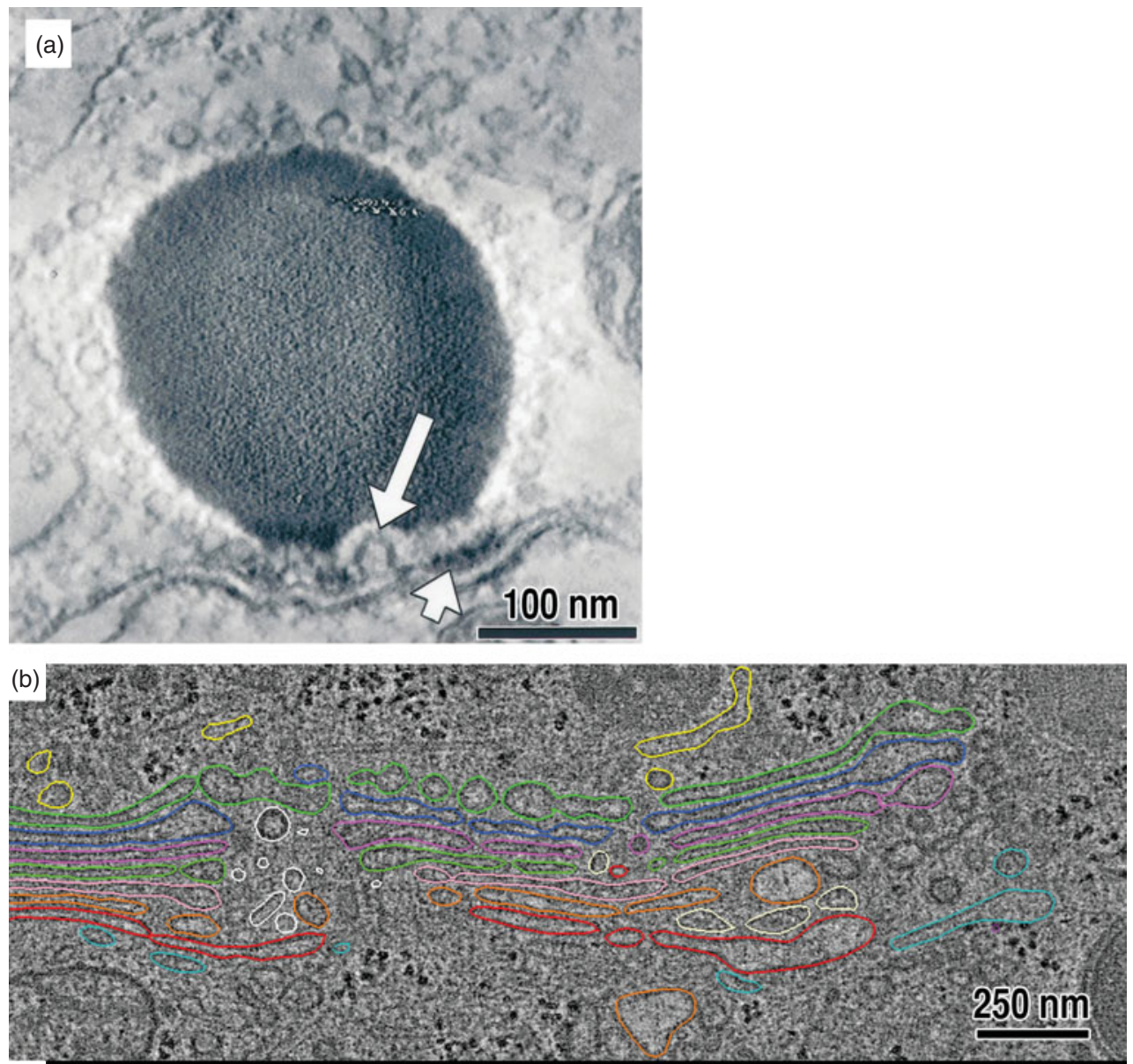

(c)

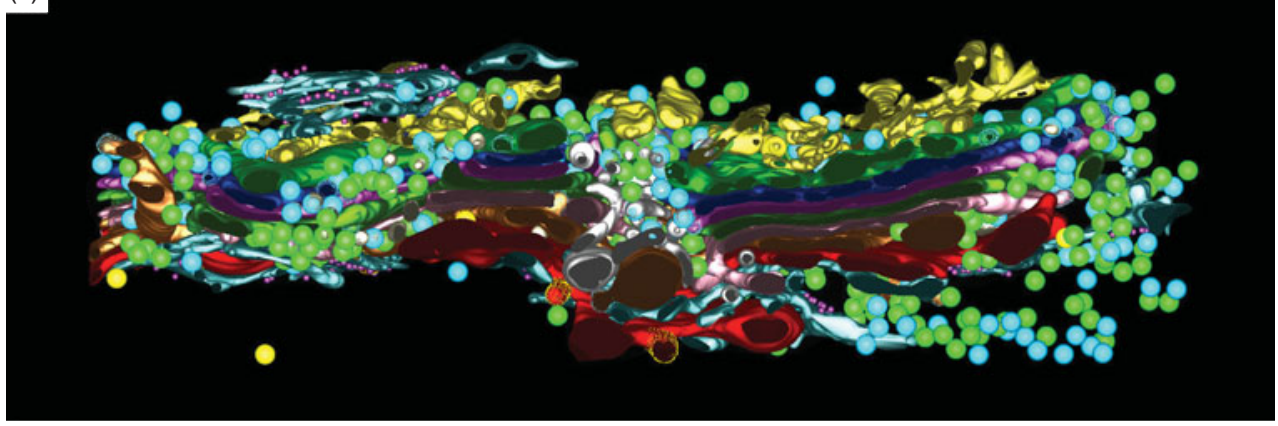

Fig. 4. Electron tomography of vesicular trafficking systems. (a) Tomographic slice of a synapse showing a synaptic body (dense round body $\sim 200 \mathrm{~nm}$ wide), $\Omega$-shaped presynaptic membrane invagination (long arrow) and presynaptic density (short arrow). (b) Tomographic slice and model (colored) of part of a Golgi body from a normal rat kidney cell. Different colors represent different cisternae. ER-Golgi intermediate compartment-yellow; trans-most cisterna - red. (c) Rendering of the model in 3D. Reproduced with permissions from (a) Lenzi et al. (1999) and (b, c) Ladinsky et al. (1999).

is the conserved nucleation site at the microtubule minus end in vivo (O’Toole et al. 1999). These tapered microtubule minus ends were also seen to be general, as they were also found in a subpopulation of spindle microtubules in the higher eukaryote C. elegans (O'Toole et al. 2003). 

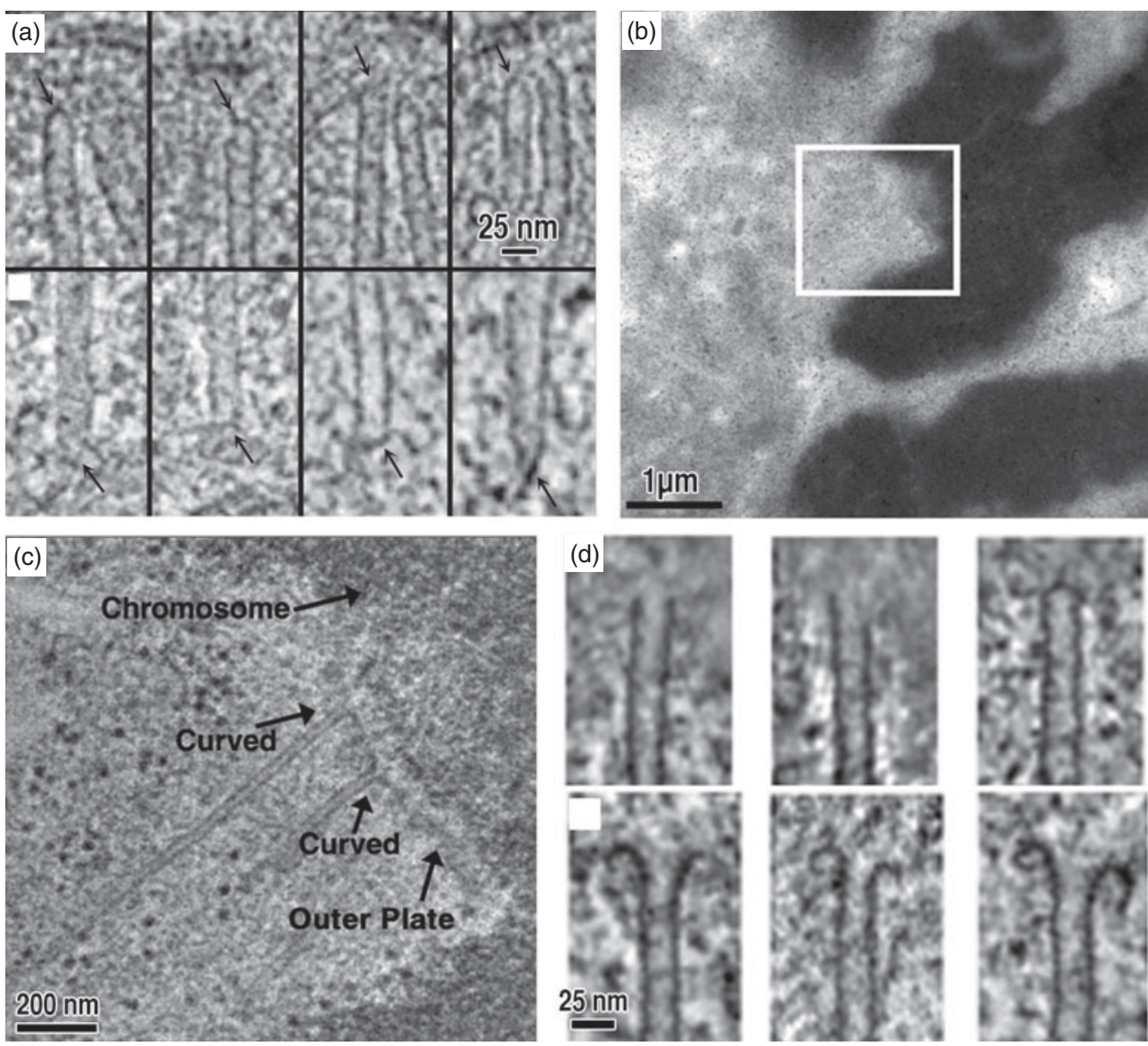

Fig. 5. Electron tomography of spindle microtubules. (a) Nuclear (upper panels) and cytoplasmic (lower panels) microtubules from tomograms of S. cerevisiae cells. (b) Low-magnification EM image of a kinetochore (boxed) from a $\mathrm{PtK}_{1}$ cell. Chromosomes are the dark, densely stained objects. (c) Tomographic slice of the region boxed in $(b)$, showing the curved morphologies of two kinetochore microtubule plus ends. (d) Gallery showing that kinetochore plus ends exhibit both blunt/straight and curved (ram's horn) morphologies. Reproduced with permissions from (a) O'Toole et al. (1999) and ( $b-d$ ) Vandenbeldt et al. (2006).

In higher eukaryotes, each chromosome attaches to the plus ends of bundled kinetochore microtubules called K-fibers. Tomograms of K-fiber microtubules showed that the protofilaments were configured into either 'flared' or 'curved' motifs, indicating that they were either growing or shrinking, respectively. Another tomographic study of individual metazoan K-fibers revealed that $2 / 3$ of the constituent kinetochore microtubules were in the depolymerizing state (Fig. $5 b-d$ ). This is surprising because the kinetochore microtubules within a single K-fiber all grow and shrink together, suggesting that the kinetochore itself must regulate the dynamics of microtubule growth and shrinkage (VandenBeldt et al. 2006). Ongoing studies seek to determine the mechanism of microtubule-kinetochore attachment (McIntosh et al. 2008).

In 2002, a seminal cryotomographic study was published on the slime mold Dictyostelium discoideum (Medalia et al. 2002). Cells were grown on EM grids, plunge-frozen, and then imaged. Since Dictyostelium cell bodies are more than $1 \mu \mathrm{m}$ thick, only their peripheries were accessible. The resulting cryotomograms revealed the cytoplasm to be densely packed with actin filaments 

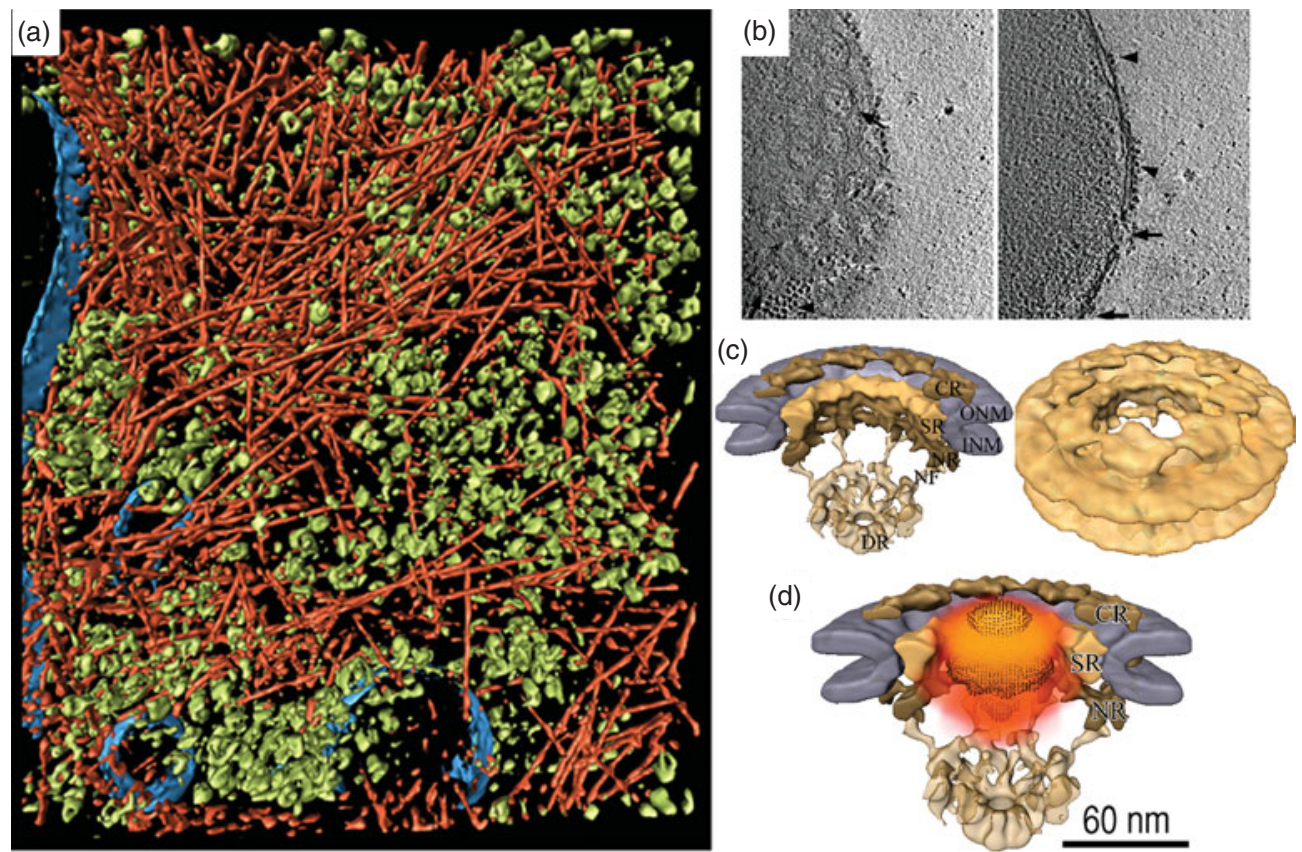

(d)

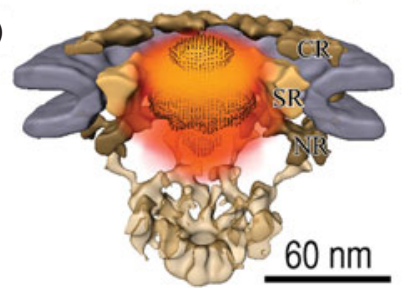

Fig. 6. Electron cryotomography of eukaryotic cells. (a) Surface rendering showing actin (red), putative ribosomes (green) and membranes (blue) at the periphery of a frozen-hydrated Dictyostelium discoideum cell. (b) Cryotomographic slices of an intact Dictyostelium nucleus showing top (left) and side (right) views of the NPC (arrows). Patches of ribosome-studded rough ER are indicated by arrowheads. (c) Subtomographic average of the NPC. (d) Probability density (orange) of gold-labeled classical import cargoes. Reproduced with permissions from (a) Medalia et al. (2002), (b) Beck et al. (2004) and (c, d) Beck et al. (2007).

and large macromolecular complexes including ribosomes and $26 \mathrm{~S}$ proteasomes (Fig. $6 \mathrm{a}$ ). Contemporary studies on the thin leading edges of cells include lamellipodia (Urban et al. 2010) and focal adhesions (Patla et al. 2010), which are both rich in actin and actin-binding proteins. The densely packed macromolecules in these cryotomograms are reminiscent of the beautiful watercolors by David Goodsell (Goodsell, 2005), and highlight how crowded cells can be (Minton, 2006).

Isolated, frozen-hydrated 'transport-competent' Dictyostelium nuclei with functional NPCs have also been imaged (Beck et al. 2004). Slices through these isolated nuclei revealed nuclear pores embedded in the nuclear envelope, which was further decorated on the outer membrane by ribosomes (Fig. 6b). Subvolumes containing these NPCs were extracted from the tomograms, aligned, and then averaged to generate a higher-resolution 'subtomographic average' of the active NPC (Fig. 6c). An elegant follow-up study showed the positions of gold-labeled test cargos, which could be mapped as a probability density cloud within the central channel of the NPC (Beck et al. 2007). The good match between the shape of the cargo density and the previously described central plug/transporter density supported the idea that the central plug/transporter is the cargo itself (Fig. 6d). Interestingly, the NPC appears to be somewhat distorted in situ, but adopts strong 8-fold rotational symmetry when purified (Yang et al. 1998). It remains to be seen if the deviation from symmetry in situ is a result of active transport, i.e., structural distortion to promote passage of the cargo molecule. These studies illustrate how tomography can be used to visualize different conformations of objects within their cellular context. 


\subsection{Tomography of tissues}

To understand the 3D ultrastructure of thicker frozen-hydrated specimens, a few groups have started to use electron cryotomography to image cryosections (Bouchet-Marquis et al. 2007; Hsieh et al. 2002). In a recent study of desmosomes (the proteinaceous intercellular junctions that adhere cells together in many tissues) for example, a human skin biopsy sample was high-pressure-frozen, cryosectioned, and imaged by electron cryotomography (Al-Amoudi et al. 2007) (Fig. 7). The cryotomograms revealed that the cadherin molecules were densely and uniformly packed, a result that differed from that of an earlier study of high-pressure-frozen/freeze-substituted skin, in which some cadherins formed discrete clusters (He et al. 2003). Subtomographic averaging of the cryotomographic densities produced an $\sim 3.4 \mathrm{~nm}$ resolution average, which was interpreted with the X-ray crystal structure of a 'classical' cadherin (Boggon et al. 2002). The quasi-atomic model of the desmosomes showed that the cadherins were highly flexible, but interacted predominantly at the midline between cells. This work represents an exciting starting point to the molecular characterization of diseases associated with malfunctioning desmosomes.

An ongoing challenge in electron microscopy is the localization and identification of proteins and protein complexes in 3D images of cells. A significant advance was made in this direction by He et al., who were able to follow immunoglobulin $\mathrm{G}(\mathrm{IgG})$ transcytosis through jejunal cells in the small intestine (He et al. 2008). In this study, neonatal rats were fed a 'milk' solution containing gold-conjugated IgG. After the IgGs were endocytosed, the rats were killed and parts of the small intestines near the jejunum were excised and immediately high-pressure-frozen. To enhance the contrast of the IgG-coupled gold tag, the intestinal tissue was treated with silver at low temperature using the same solvents needed for the subsequent FS step (He et al. 2007; Morphew et al. 2008). The tissue was then subjected to FS, plastic embedding and microtomy. Tomograms from the resulting plastic sections clearly showed tags in a diversity of vesicles and between cells in the lateral intercellular space (Fig. 8). Further development of this experimental system may yield a complete $\operatorname{IgG}$ molecular-trafficking map from the apical to the basolateral side of an entire cell.

\subsection{Bacterial ultrastructure}

Filaments were so rarely seen in traditional thin-section EM images of bacteria that their absence was long thought to be one of the distinguishing characteristics of 'prokaryotes'. The advent of higher-resolution fluorescence light microscopes and GFP-tagging methods then revolutionized bacterial cell biology by suggesting that numerous bacterial proteins form helices, rings and other filaments (Gitai, 2005). Electron cryotomography has proven essential in clarifying, adding to, and in some cases refuting these claims.

Cytoskeletal filaments were first directly visualized by electron cryotomography in the thin spiral-shaped bacterium Spiroplasma melliferum (Kurner et al. 2005). In the classic model species C. crescentus, multiple classes of filaments including 'cytoplasmic', 'ring-like', 'polar' and 'inner curvature' were then discovered (Briegel et al. 2006). The latter class of filaments (Fig. 9 a) were later shown by correlated LM-electron cryotomography experiments to be polymers of CTP synthase that negatively regulated cell curvature (Ingerson-Mahar et al. 2010). More filaments were discovered by cryotomography in the Gram-negative magnetotactic bacterium Magnetospirillum magneticum, this time flanking linear arrays of magnetosomes (a bacterial 'organelle' that contains a magnetite crystal and aligns the cell to the geomagnetic field) (Komeili et al. 2006; 

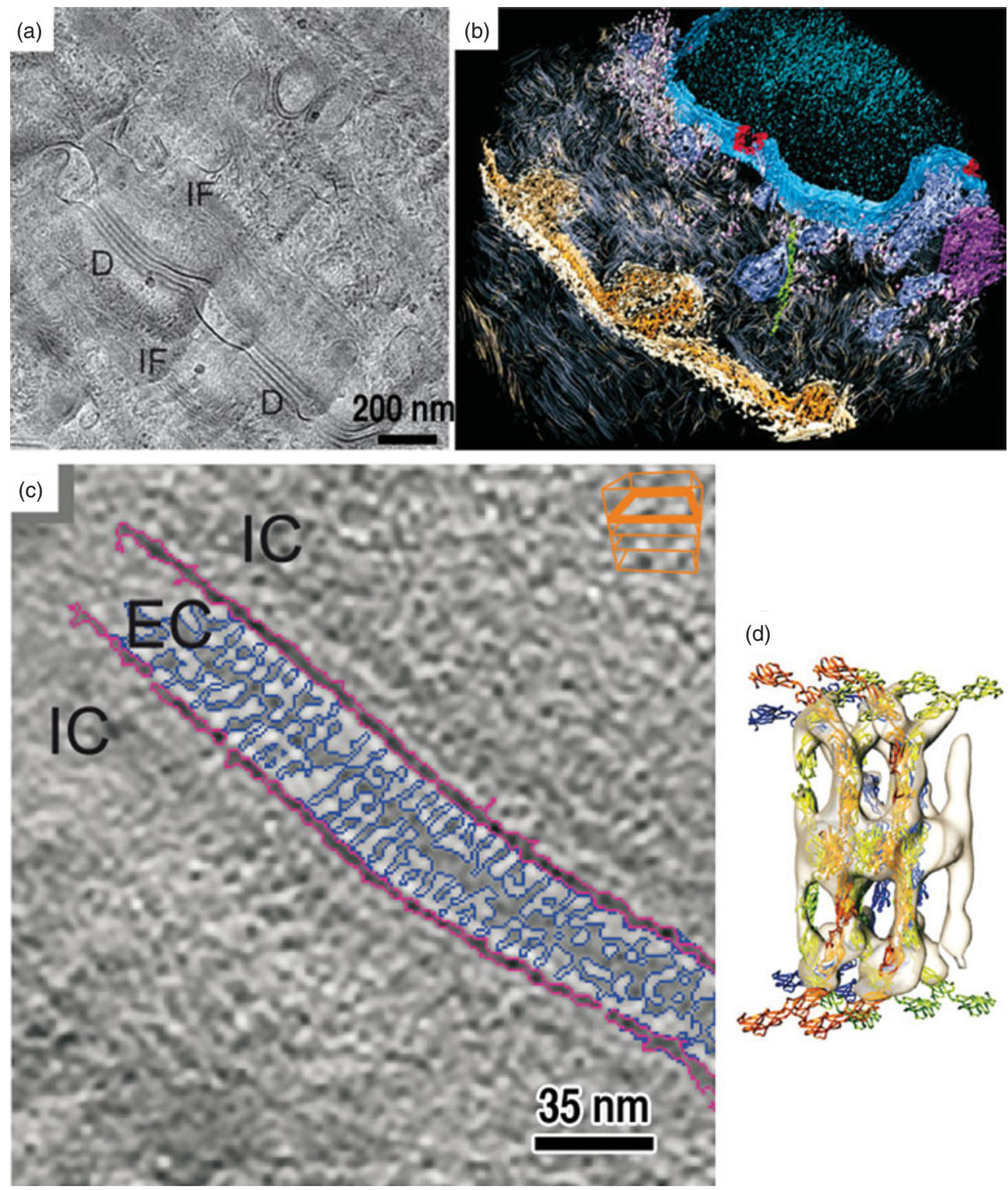

Fig. 7. Electron cryotomography of vitreous tissue sections. (a) Cryo-EM image of a cryosectioned human skin biopsy showing desmosomes (D) and filament networks (IF). (b) Isosurface rendering of a cryotomogram from another region of skin showing desmosomes (gold/brown), the nucleus (blue), nuclear pores (red) and a putative microtubule (green). (c) Cryotomographic slice of a desmosome showing extracellular (EC) and intracellular (IC) spaces. (d) Subtomographic average of C-cadherin molecules (white isosurfaces) with crystal structures docked. Reproduced with permission from Al-Amoudi et al. (2007).

Scheffel et al. 2006). These filaments were identified as the actin-like protein MamK. The cryotomograms also delivered an unexpected surprise: the magnetosomes were not true organelles in which the membranes formed closed vesicles, but were instead simply invaginations of the inner membrane. A second bacterial actin homolog, ParM, was seen forming bundles of 

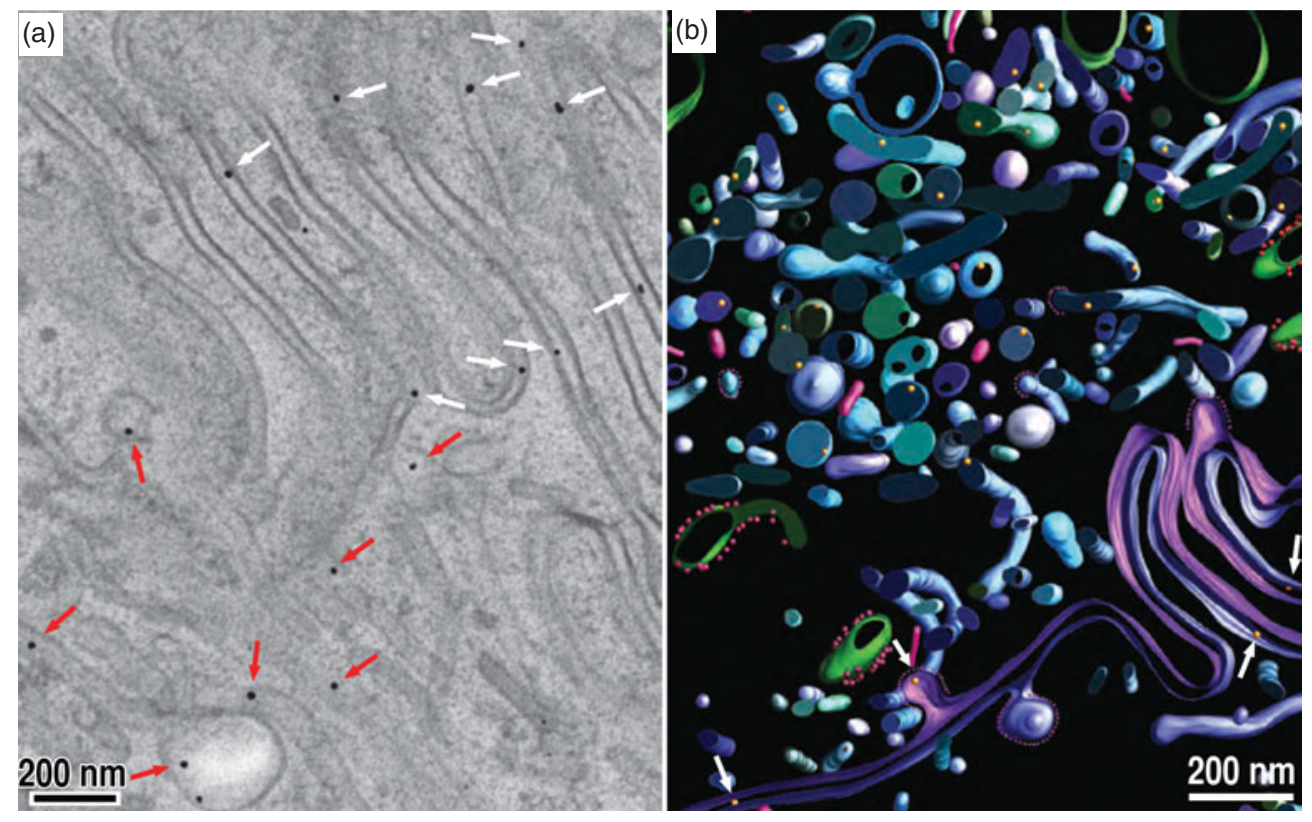

Fig. 8. Transcytosis followed in tissues. (a) Tomographic slice of the lateral intercellular space (LIS) between two neonatal rat jejunal cells. Gold-labeled Fc (Au-Fc) molecules can be found in both the LIS (red arrows) and irregular vesicles (white arrows). (b) 3D segmentation of the region in (a) showing membranes and vesicles in green and blue, and Au-Fc in gold. Reproduced with permission from He et al. (2008).

filaments in situ, clarifying the number and arrangement of filaments in these plasmid-segregating machines (Salje et al. 2009). Filaments of the tubulin-like FtsZ protein were visualized in dividing C. crescentus cells ( $\mathrm{Li}$ et al. 2007). Instead of complete rings as expected from fluorescence light microscopy, FtsZ was shown to form short arc-like fragments adjacent to the inner membrane of the constriction site. The filaments adopted both straight and curved conformations, which supported an 'iterative pinching' model for cell constriction (Erickson, 1997).

It is noteworthy that magnetosomes and dividing bacterial cells had been thoroughly studied using traditional EM, but the MamK and FtsZ filaments were never detected, probably because the chemical fixation and dehydration caused them to depolymerize (Pilhofer et al. 2010). In contrast, certain filaments that were thought to exist based on fluorescence microscopy have not been detected in cryotomograms. The actin homolog MreB, for instance, was reported to form long helices surrounding rod-shaped cells just inside their cytoplasmic membranes (Chiu et al. 2008; Figge et al. 2004; Jones et al. 2001; Shih et al. 2003; Srivastava et al. 2007). Puzzlingly, no such helices were ever seen in cryotomograms (Swulius et al. 2011). New fluorescence images are now showing the helical patterns seen previously by light microscopy were apparently just artifacts (Dominguez-Escobar et al. 2011; Garner et al. 2011).

Electron cryotomography has also been instrumental in the study of megadalton-sized bacterial molecular machines in their native context. This is especially true for the bacterial flagellar motor, which cannot be purified intact because it loses the torque-generating 'stator' component (Tang et al. 1996; Thomas et al. 2001). Intact flagellar motors were first visualized in cryotomograms of the spirochaete Treponema primitia, a bacterium with periplasmic flagella 

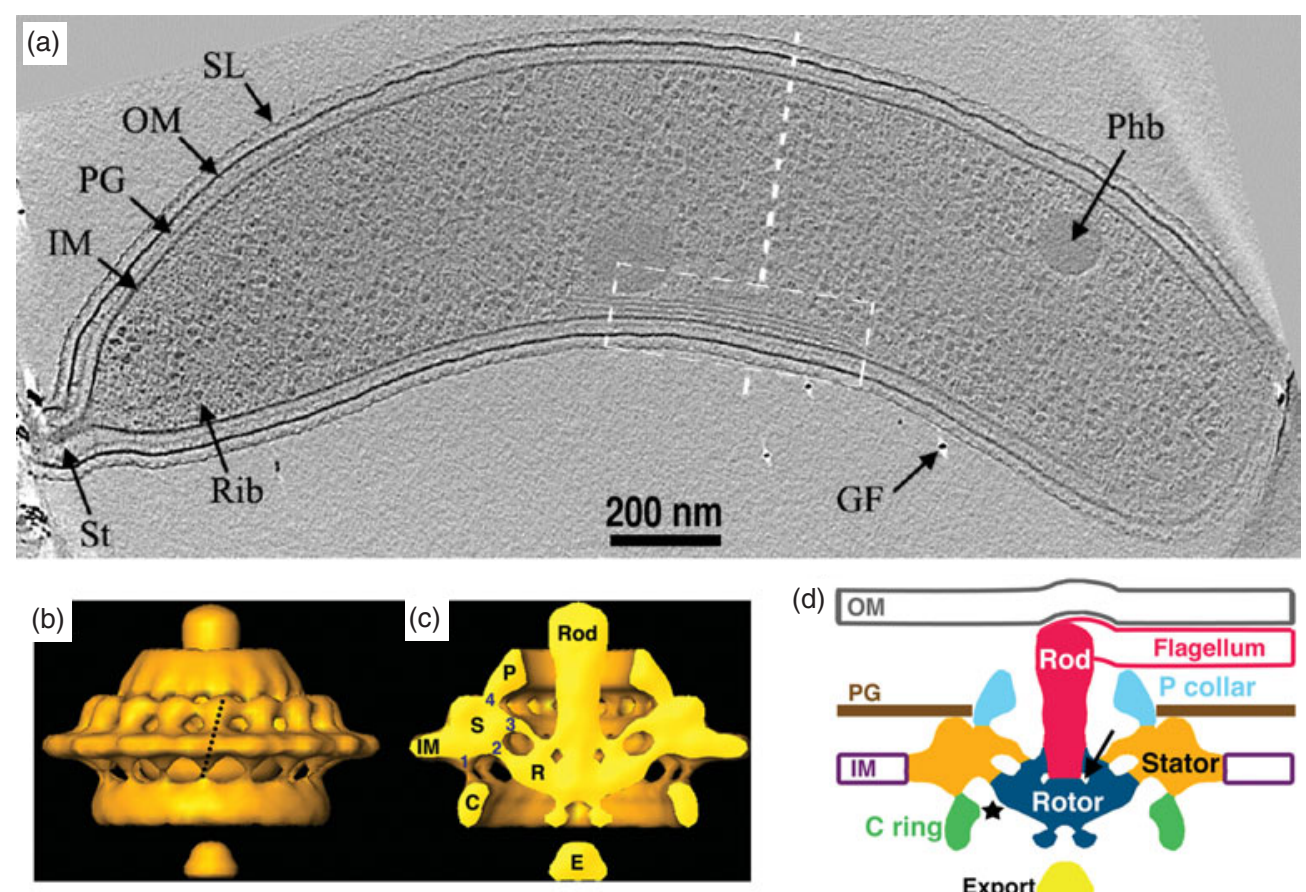

(d)

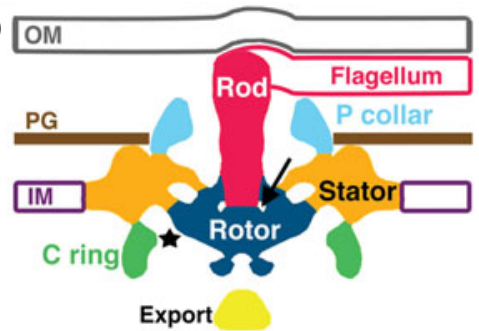

Fig. 9. Insights into bacterial ultrastructure. (a) Cryotomographic slice of a Caulobacter crescentus cell showing the inner curvature filaments (white dashed box). Other features include the S-layer (SL), outer membrane $(\mathrm{OM})$, peptidoglycan layer (PG), inner membrane (IM), stalk (St), putative ribosomes (Rib), putative poly$\beta$-hydroxybutyrate granule (Phb) and gold fiducial (GF). (b) Isosurface rendering of a Treponema primitia flagellar motor subtomographic average. (c) Cutaway of $(b)$, with components labeled: peptidoglycanbinding collar $(\mathrm{P})$, stator $(\mathrm{S})$, rotor $(\mathrm{R})$, cytoplasmic ring $(\mathrm{C})$, putative export structure $(\mathrm{E})$ and inner membrane (IM). (d) Cartoon of the motor's organization based on the cryotomography data. Reproduced with permissions from Briegel et al. (2006)and Murphy et al. (2006).

(Murphy et al. 2006). The motor was then reconstructed to $\sim 7 \mathrm{~nm}$ resolution by subtomographic averaging and the imposition of 16 -fold rotational symmetry (Fig. $9 b-d$ ). In contrast to the flagellar motors of other bacteria, the Treponema motor is unusually wide, suggesting how it might generate the higher levels of torque needed to rotate the flagellum within the periplasm (Murphy et al. 2008). Indeed, flagellar motors from the Borrelia spirochaetes shared a similar architecture (Kudryashev et al. 2010; Liu et al. 2009). A recent cryotomographic comparison of 11 different motors has highlighted how this amazing molecular machine has evolved to meet the needs of different species (Chen et al. 2011).

Subtomographic averaging has also furthered our understanding of bacterial chemotaxis. Bacteria sense molecules in their environment and regulate the direction of their swimming using an array of chemoreceptors embedded in the inner membrane. These arrays are exquisitely sensitive, and can dynamically adjust their response to a staggering five orders-of-magnitude range of attractant concentrations (Alon et al. 1999). While X-ray crystallography and NMR spectroscopy have delivered atomic models of various components of this signaling system, cryotomography showed that the receptors are universally organized in vivo in hexagonal arrays of trimers-of-receptor-dimers (Briegel et al. 2008, 2009; Khursigara et al. 2008 a, b), providing a basis for mechanistic models of the cooperativity. 

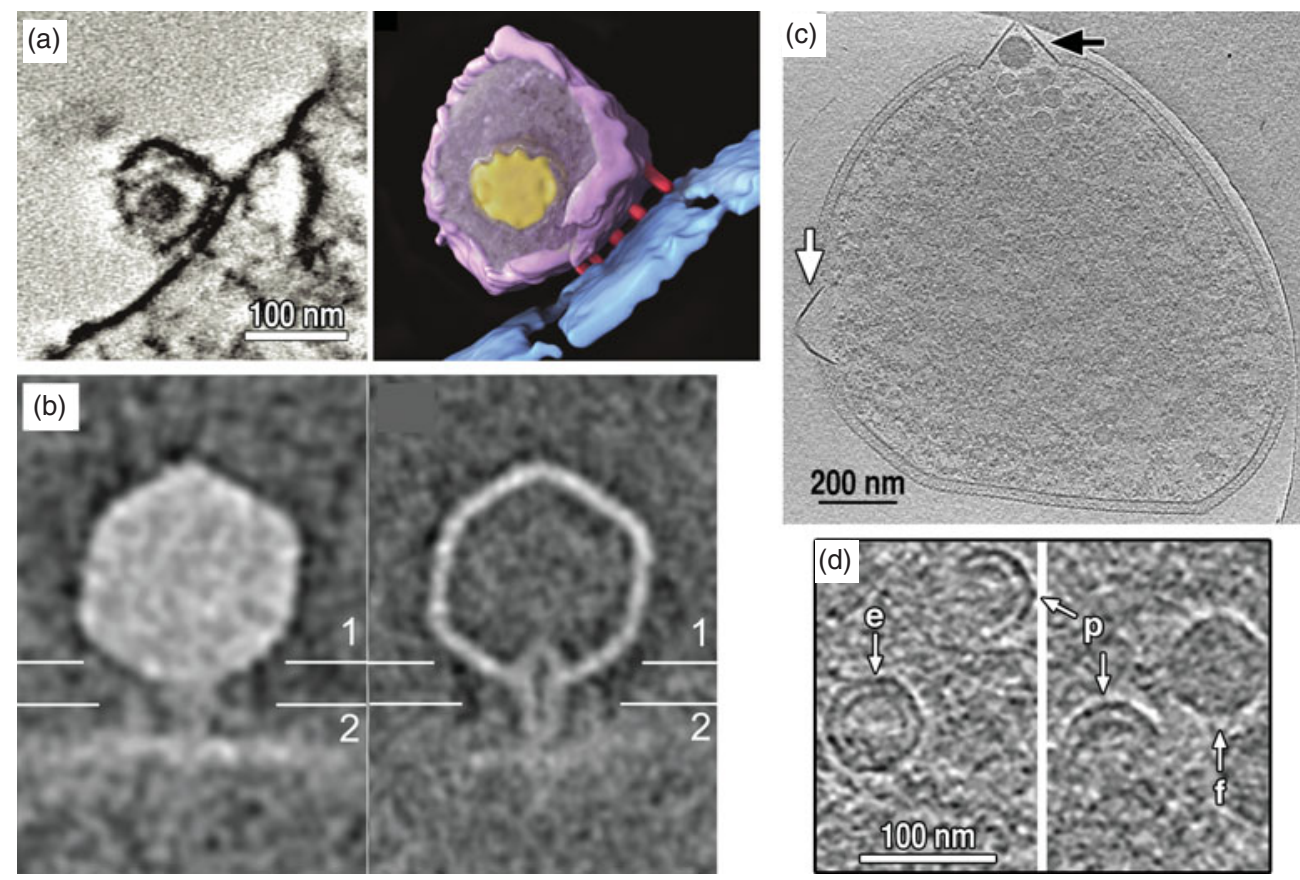

Fig. 10. Virus-cell interactions. (a) Tomographic slice (left) and surface rendering (right) of HIV-1 bound to a T-cell. Structures are colored as: viral membrane (purple), core (yellow), cell membrane (blue) and 'entry claw' contacts (red). (b) Central slices through the subtomographic averages of full (left) and emptied (right) $\varepsilon 15$ bacteriophages bound to Salmonella hosts. (c) Cryotomographic slice of a Sulfolobus solfataricus cell, infected with Sulfolobus turreted icosahedral virus (STIV). Virus-induced pyramids appeared with electrondense bodies (black arrow) or without supermolecular complexes (white arrow). (d) Cryotomographic slices of partially assembled (p), empty $(e)$ and filled $(f)$ STIV. Reproduced with permissions from Sougrat $e t$ al. (2007), Fu et al. (2010) and Chang et al. (2010).

\subsection{Virus-cell interactions}

While cryo-EM single particle analysis has been used to study symmetric viral capsids (Liu et al. 2010; Wolf et al. 2010), tomography has been needed to understand asymmetric viral particles like Herpesvirus (Grunewald et al. 2003), HIV (Benjamin et al. 2005; Briggs et al. 2006; Wright et al. 2007), influenza (Harris et al. 2006) and bunyaviruses (Overby et al. 2008). In addition, tomography has proven vital to visualize the interactions of viruses with their cellular hosts, both during infection and assembly/egress. The following three recent tomographic studies, taken from the eukaryotic, bacterial and archael domains of life, respectively, are good examples.

In a study of Simian immunodeficiency virus (SIV) and HIV-1 entry, viruses were incubated briefly with cell lines expressing the appropriate receptors and co-receptors (Sougrat et al. 2007). The cell/virus infection mixture was then fixed, plastic-embedded, sectioned and imaged. Tomograms showed that both SIV and HIV engaged their target cells with an 'entry claw' (Fig. 10 a) that apparently concentrates virus glycoprotein spikes and possibly host receptors in a small patch of 5-7 large densities. Surprisingly, glycoprotein spikes were largely absent from the non-interacting hemisphere of the virion. Further work using this retrovirus infection system should help resolve longstanding questions about what conformational changes drive cell-virus membrane fusion (White et al. 2010). 
A different study investigated the conformational changes that dsDNA bacteriophage undergo when infecting their bacterial hosts (Chang et al. 2010). Phage $\varepsilon 15$ were mixed with Salmonella and sites where phage had docked onto the host cell were imaged with electron cryotomography (Fig. 10 b). Some phages exhibited DNA-filled heads whereas other phages had already injected their genomes and were therefore empty. A comparison of the full and empty phage showed that the tail must undergo major conformational changes to inject the viral genome. The tail hub extends a tube that spans the entire periplasm, presumably protecting the viral DNA from degradation. In the emptied phage, the tail's interior channel appears tapered, possibly to seal up the injection site.

Archael viruses can drastically alter the structure of their hosts, as illustrated by a 3D study of the last stages of infection (Fu et al. 2010). Here, Sulfolobus cells were infected with the Sulfolobus turreted icosahedral virus, a dsDNA virus of the Adeno-PRD1 lineage that has an internal lipid membrane layer (Khayat et al. 2005). After the infection had progressed for many hours, Sulfolobus cells were plunge-frozen and imaged by electron cryotomography. The cryotomograms showed new details about the pyramid structures that protrude from the cell surface (Brumfield et al. 2009) (Fig. 10 c). In the cytoplasm, some partially assembled viruses were discovered, revealing a partial capsid protein shell and a partial lipid layer (Fig. 10d). This unexpected finding suggests that in Sulfolobus turreted icosahedral virus (STIV) and related viruses, the capsid and lipid membrane co-assemble in the cytoplasm. This de novo assembly of viral membrane is especially intriguing because uninfected Sulfolobus cells apparently do not assemble cytoplasmic vesicles or organelles. Much of the early (initial infection) and terminal (lysis) stages of the STIV lifecycle remain to be characterized.

\section{Future developments}

Given electron tomography's ability to reveal the ultrastructure of cells and other unique biological objects in $3 \mathrm{D}$ to macromolecular resolution, it is likely to have a long and powerful impact on cell biology. The technique is still young, however, and so there are many opportunities for improvement.

\section{I Better specimens}

The quality of any image is limited by the quality of the specimen. Unfortunately, since only slender bacterial and archaeal cells and the thin edges of some eukaryotic cells are thin enough to image directly (see above), the majority of eukaryotic cells and many bacterial and archaeal cells must be sectioned. While this is straightforward to do after plastic-embedding, efforts are underway to develop techniques to allow the interiors of these cells to be imaged in the more lifelike, frozen-hydrated state. While thin vitreous sections can be produced by cryoultramicrotomy (Al-Amoudi et al. 2004), this technique generates low yields of good specimens. In a recent study that highlights this technology's promise, however, hundreds of tilt series were recorded from cryosection ribbons of the picoplankton Ostreococcus tauri, a tiny eukaryote just $\sim 0.5-1 \mu \mathrm{m}$ thick (Gan et al. 2011; Henderson et al. 2007). Individual microtubule protofilaments could be resolved in the vitreous ice deep within the nucleus, and revealed the surprising result that $O$. tauri segregates its genome with fewer microtubules than chromosomes. Technologies such as micromanipulator-assisted handling of the elongating cryosection (Ladinsky et al. 2006), electrostatic charging of the cryosection to make it adhere to the grid without the use of mechanical 
stamping (Pierson et al. 2010), and the solubilization of quantum dots or gold colloids in cryogenic organic solvents for deposition onto cryosections (Gruska et al. 2008; Masich et al. 2006) may improve the yield in the future.

As mentioned above, cryosections today also suffer from compression along the cutting direction and crevasses and knife marks on the two faces (Dubochet et al. 2007). Crevasses can be somewhat reduced when cryosections are cut just $20 \mathrm{~nm}$ thick (Zhang et al. 2004), but this severely reduces the cellular volume sampled in each tomogram. Recently, the cryo focused-ionbeam (FIB) milling method was developed as a way to produce cryosections with minimal 'cutting' artifacts (Hayles et al. 2010; Marko et al. 2007; Rigort et al. 2010). Here, a beam of ions (typically Gallium) is used to thin a pellet of high-pressure-frozen cells. Because there is no mechanical cutting, there are fewer surface artifacts. FIB milling can only generate one cryosection per experiment, however, prohibiting serial sectioning. As with traditional plasticembedding, and then FS, advances such as plunge-freezing and cryosectioning have revealed new details and caused some earlier images to be reinterpreted. Thus, while we have certainly been moving towards more native specimens, history reminds us to remain cautious about our conclusions no matter how advanced our methods are.

\subsection{Better images}

Cryo-EM images have low contrast in part because the phase CTF has a sinusoidal shape and starts with zero contrast transfer at zero spatial frequency. There is a small amount of cosine-like amplitude contrast, but its contribution from light elements like carbon, nitrogen and oxygen is small. Low-resolution phase contrast can be increased substantially by using a phase plate (Nagayama \& Danev, 2009). As in phase-contrast light microscopy, the scattered beam is made to undergo a phase shift relative to the unscattered beam either by passing through a thin amorphous carbon film (Danev \& Nagayama, 2008) or an electric field (Cambie et al. 2007; Majorovits et al. 2007). The unscattered and scattered electrons then recombine at the image plane to form a higher contrast image generated through a cosine-like CTF. Images from the first generation of phase plates show impressive increases in low frequency contrast (Fig. 11), but it remains to be seen whether any new high-resolution details are also recoverable. If a spherical-aberration corrector and a phase plate were combined, the oscillations of the CTF and the challenges of the defocus gradient could be nearly eliminated in the resolution range of interest in electron tomography (Danev et al. 2009).

Current phosphor-CCD detectors suffer from large point spread functions and high noise from the electron-photon conversion process. A number of groups have now made progress designing and fabricating 'direct' detectors, semiconductor devices that generate signal from the impact of the high-energy imaging electrons themselves (Deptuch et al. 2007; McMullan et al. 2007; Milazzo et al. 2005). Direct detectors should also read-out quickly enough to discriminate individual electron hits, allowing each imaging electron to be counted with equal weight. These two factors will dramatically improve the modulation transfer function (McMullan et al. 2009), resulting in a nearly perfect detector.

\subsection{Better analyses}

The most time-consuming step in electron tomography today is the analysis of the tomograms. More powerful and automatic methods to calculate, refine, denoise, segment and search 

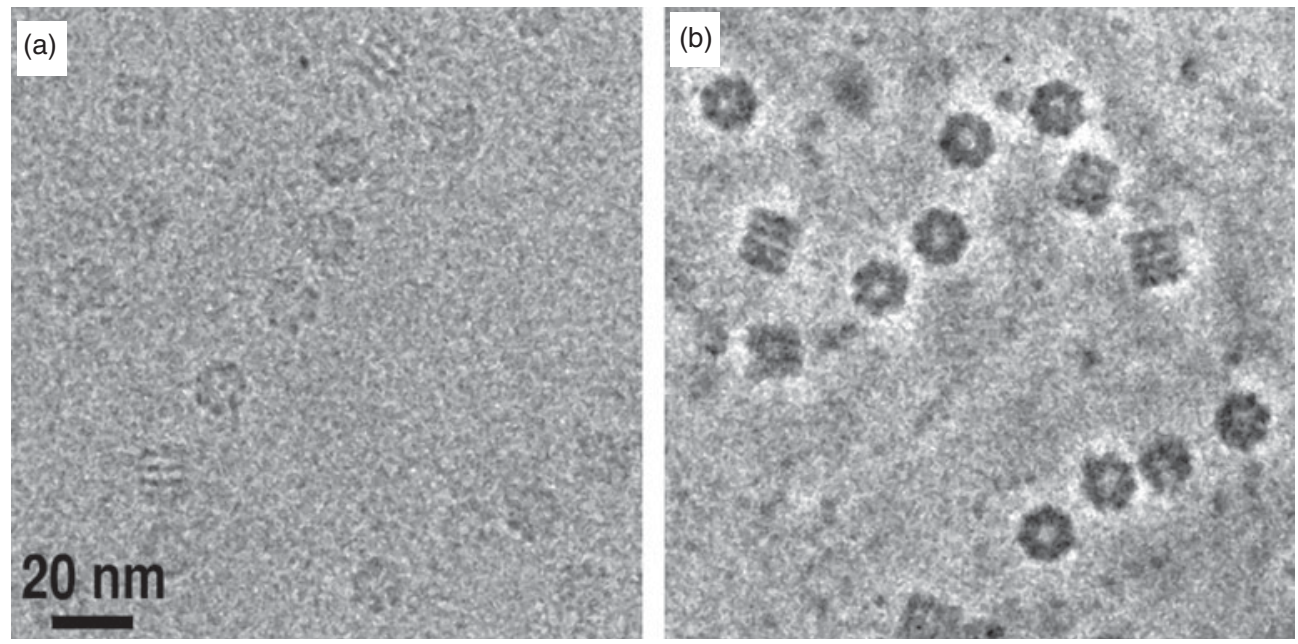

Fig. 11. Zernike phase-contrast. Cryo-EM of GroEL with (a) defocus phase contrast and (b) Zernike phase contrast generated by a thin amorphous-carbon phase plate. Reproduced with permission from Danev \& Nagayama (2008).

tomograms are needed. To identify proteins of interest, high contrast genetically encodable tags are being developed. The metal-binding protein metallothionein appears in vitro to be an attractive candidate (Mercogliano \& DeRosier, 2007), though it remains to be seen if metallothionein-gold tags can be localized in 3D electron cryotomograms of cells and how broadly this tag can be applied (Diestra et al. 2009). Ferritin-based tags may also be used to localize proteins, as recently demonstrated in cryotomograms of bacterial cells (Wang et al. 2011). Another promising tagging strategy for plastic-embedded samples is the ReAsh-diaminobenzadine (ReAsh-DAB) system, which allows co-localization of ReAsh in fluorescence images and DAB-nucleated osmium haloes in EM images (Gaietta et al. 2002). It remains to be seen, however, how precisely the $\mathrm{DAB}$-osmium halo could localize a smaller object in $3 \mathrm{D}$ in a tomogram.

While the real power of tomography is its ability to produce a $3 \mathrm{D}$ image of a single unique object, when there are multiple copies of a particular structure in a cell or a group of cells, additional information can be gained by classification and averaging. New and better tools (both software and hardware) for this are needed. As an example, the maximum-likelihood method (Sigworth, 1998) promises to improve the averaging of particles from cellular environments, where many will exhibit conformational heterogeneity and differences in assembly state. Classification and alignment of subtomograms are done simultaneously and the missing wedge is treated as unobserved data (Scheres et al. 2009). The reconstructions are generated in iterations in which a weighted average of all possible alignments and class assignments contributes to the reconstruction in each cycle. Since the maximum-likelihood method is robust to missing data, noisy data, and conformational heterogeneity, it can produce model-bias-free subtomographic reconstructions, but it is computationally demanding (Fu et al. 2010; Ortiz et al. 2010).

\section{Conclusion}

Electron tomography has begun to deliver 3D images of cells with sufficient resolution to visualize the structure of large macromolecular complexes and their arrangement in situ. Such 
images are vital, since in many cases structures of interest only assemble or only exhibit functional conformations in their cellular environment. Usually, tomographic imaging leads directly to mechanistic hypotheses. Thus while the contributions of electron tomography to eukaryotic, bacterial and archael cell biology have already been substantial (see examples above), we believe it is just the beginning. Improved methods for serial cryosectioning and labeling will dramatically expand the utility of electron tomography in coming years. EMs capable of tomography are still rare, expensive and frequently out of service, but many institutions are now investing in these instruments and new research groups are rapidly sprouting up around the world with the expertise to exploit them. The development of direct detectors, phase plates and aberration correctors will dramatically improve the information content of future tilt series, and both the software tools and the raw computational power needed to extract structural details from the tomograms are increasing. Electron tomograms will increasingly be the 'place' where sequencing 'parts lists', atomic models, biochemical measurements and single-molecule biophysical data meet to reveal mechanism.

\section{Acknowledgements}

We thank Morgan Beeby, Ariane Briegel, Mark Ladinsky, Alasdair McDowall and Martin Pilhofer for comments and Karin Mallard for help with figures and editing. We apologize to all our colleagues whose work we could not highlight for space constraints. This work was supported in part by NIH grants GM066521 and GM094800B to G. J. J.

\section{References}

Adrian, M., Dubochet, J., Lepault, J. \& Mcdowall, A. W. (1984). Cryo-electron microscopy of viruses. Nature 308, 32-36.

Al-Amoudi, A., Chang, J. J., Leforestier, A., Mcdowall, A., Salamin, L. M., Norlen, L. P., Richter, K., Blanc, N. S., Studer, D. \& Dubochet, J. (2004). Cryo-electron microscopy of vitreous sections. EMBO Journal 23, 3583-3588.

Al-Amoudi, A., Diez, D. C., Betts, M. J. \& Frangakis, A. S. (2007). The molecular architecture of cadherins in native epidermal desmosomes. Nature 450, 832-837.

Alon, U., Surette, M. G., Barkai, N. \& Leibler, S. (1999). Robustness in bacterial chemotaxis. Nature 397, 168-171.

Amat, F., Moussavi, F., Comolli, L. R., Elidan, G., Downing, K. H. \& Horowitz, M. (2008). Markov random field based automatic image alignment for electron tomography. Journal of Structural Biology 161, 260-275.

Beck, M., Forster, F., Ecke, M., Plitzko, J. M., Melchior, F., Gerisch, G., Baumeister, W. \& Medalia, O. (2004). Nuclear pore complex structure and dynamics revealed by cryoelectron tomography. Science 306, 1387-1390.

Beck, M., Lucic, V., Forster, F., Baumeister, W. \& Medalia, O. (2007). Snapshots of nuclear pore

complexes in action captured by cryo-electron tomography. Nature 449, 611-615.

Beck, M., Malmstrom, J. A., Lange, V., Schmidt, A., Deutsch, E. W. \& Aebersold, R. (2009). Visual proteomics of the human pathogen Leptospira interrogans. Nature Methods 6, 817-823.

Belmont, A. S., Sedat, J. W. \& Agard, D. A. (1987). A three-dimensional approach to mitotic chromosome structure: evidence for a complex hierarchical organization. Journal of Cell Biology 105, 77-92.

Benjamin, J., Ganser-Pornillos, B. K., Tivol, W. F., Sundquist, W. I. \& Jensen, G. J. (2005). Threedimensional structure of HIV-1 virus-like particles by electron cryotomography. Journal of Molecular Biology 346, 577-588.

Bennett, P. M. (1974). Decrease in section thickness on exposure to the electron beam; the use of tilted sections in estimating the amount of shrinkage. Journal of Cell Science 15(3), 693-701.

Boggon, T. J., Murray, J., Chappuis-Flament, S., Wong, E., Gumbiner, B. M. \& Shapiro, L. (2002). C-cadherin ectodomain structure and implications for cell adhesion mechanisms. Science 296, 1308-1313.

Bottcher, B., Wynne, S. A. \& Crowther, R. A. (1997). Determination of the fold of the core protein of 
hepatitis B virus by electron cryomicroscopy. Nature 386, 88-91.

Bouchet-Marquis, C., Zuber, B., Glynn, A. M., Eltsov, M., Grabenbauer, M., Goldie, K. N., Thomas, D., Frangakis, A. S., Dubochet, J. \& Chretien, D. (2007). Visualization of cell microtubules in their native state. Biology of the Cell 99, 45-53.

Brandt, F., Carlson, L. A., Hartl, F. U., Baumeister, W. \& Grunewald, K. (2010). The three-dimensional organization of polyribosomes in intact human cells. Molecular Cell 39, 560-569.

Briegel, A., Dias, D. P., Li, Z., Jensen, R. B., Frangakis, A. S. \& Jensen, G. J. (2006). Multiple large filament bundles observed in Caulobacter crescentus by electron cryotomography. Molecular Microbiology 62, 5-14.

Briegel, A., Ding, H. J., Li, Z., Werner, J., Gitai, Z., Dias, D. P., Jensen, R. B. \& Jensen, G. J. (2008). Location and architecture of the Caulobacter crescentus chemoreceptor array. Molecular Microbiology 69, 30-41.

Briegel, A., Ortega, D. R., Tocheva, E. I., Wuichet, K., Li, Z., Chen, S., Muller, A., Iancu, C. V., Murphy, G. E., Dobro, M. J., Zhulin, I. B. \& Jensen, G. J. (2009). Universal architecture of bacterial chemoreceptor arrays. Proceedings of the National Academy of Sciences of the United States of America 106(40), 17181-17186.

Briggs, J. A., Grunewald, K., Glass, B., Forster, F., Krausslich, H. G. \& Fuller, S. D. (2006). The mechanism of HIV-1 core assembly: insights from three-dimensional reconstructions of authentic virions. Structure 14, 15-20.

Brumfield, S. K., Ortmann, A. C., Ruigrok, V., Suci, P., Douglas, T. \& Young, M. J. (2009). Particle assembly and ultrastructural features associated with replication of the lytic archaeal virus sulfolobus turreted icosahedral virus. Journal of Virology 83, 5964-5970.

Cambie, R., Downing, K. H., Typke, D., Glaeser, R. M. \& Jin, J. (2007). Design of a microfabricated, twoelectrode phase-contrast element suitable for electron microscopy. Ultramicroscopy 107(4-5), 329-339.

Chang, J. T., Schmid, M. F., Haase-Pettingell, C., Weigele, P. R., King, J. A. \& Chiu, W. (2010). Visualizing the structural changes of bacteriophage Epsilon15 and its Salmonella host during infection. Journal of Molecular Biology 402, 731-740.

Chen, S., Beeby, M., Murphy, G. E., Leadbetter, J. R., Hendrixson, D. R., Briegel, A., Li, Z., Shi, J., Tocheva, E. I., Muller, A., Dobro, M. J. \& Jensen, G. J. (2011). Structural diversity of bacterial flagellar motors. EMBO Journal 30, 2972-2981.

Chen, S., Mcdowall, A., Dobro, M. J., Briegel, A., Ladinsky, M., Shi, J., Tocheva, E. I., Beeby, M., Pilhofer, M., Ding, H. J., Li, Z., Gan, L., Morris, D. M. \& Jensen, G. J. (2010). Electron cryotomography of bacterial cells. Journal of Visualized Experiments (39), e1943.
Chiu, S. W., Chen, S. Y. \& Wong, H. C. (2008). Dynamic localization of MreB in Vibrio parahaemolyticus and in the ectopic host bacterium Escherichia coli. Applied and Environmental Microbiology 74, 6739-6745.

Chretien, D., Fuller, S. D. \& Karsenti, E. (1995). Structure of growing microtubule ends: twodimensional sheets close into tubes at variable rates. Journal of Cell Biology 129, 1311-1328.

Clermont, Y., Rambourg, A. \& Hermo, L. (1995). Trans-Golgi network (TGN) of different cell types: three-dimensional structural characteristics and variability. Anatomical Record 242, 289-301.

Conway, J. F., Cheng, N., Zlotnick, A., Wingfield, P. T., Stahl, S. J. \& Steven, A. C. (1997). Visualization of a 4-helix bundle in the hepatitis B virus capsid by cryo-electron microscopy. Nature 386, 91-94.

Crowther, R. A., Derosier, D. J. \& KLug, A. (1970). The reconstruction of a three-dimensional structure from projections and its application to electron microscopy. Proceedings of the Royal Society of London. Series A, Mathematical and Physical Sciences 317, 319-340.

Danev, R., Glaeser, R. M. \& Nagayama, K. (2009). Practical factors affecting the performance of a thin-film phase plate for transmission electron microscopy. Ultramicroscopy 109, 312-325.

Danev, R. \& Nagayama, K. (2008). Single particle analysis based on Zernike phase contrast transmission electron microscopy. Journal of Structural Biology 161, 211-218.

Deptuch, G., Besson, A., Rehak, P., Szelezniak, M., Wall, J., Winter, M. \& Zhu, Y. (2007). Direct electron imaging in electron microscopy with monolithic active pixel sensors. Ultramicroscopy 107, 674-684.

Diestra, E., Fontana, J., Guichard, P., Marco, S. \& Risco, C. (2009). Visualization of proteins in intact cells with a clonable tag for electron microscopy. Journal of Structural Biology 165, 157-168.

Ding, R., Mcdonald, K. L. \& Mcintosh, J. R. (1993). Three-dimensional reconstruction and analysis of mitotic spindles from the yeast, Schizosaccharomyces pombe. Journal of Cell Biology 120, 141-151.

Dominguez-Escobar, J., Chastanet, A., Crevenna, A. H., Fromion, V., Wedlich-Soldner, R. \& Carballido-Lopez, R. (2011). Processive movement of MreB-associated cell wall biosynthetic complexes in bacteria. Science 333, 225-228.

Dubochet, J., Adrian, M., Chang, J.J., Homo, J. C., Lepault, J., Mcdowall, A. W. \& Schultz, P. (1988). Cryo-electron microscopy of vitrified specimens. Quarterly Review of Biophysics 21, 129-228.

Dubochet, J., Mcdowall, A. W., Menge, B., Schmid, E. N. \& Lickfeld, K. G. (1983). Electron microscopy of frozen-hydrated bacteria. Journal of Bacteriology 155, 381-390.

Dubochet, J., Zuber, B., Eltsov, M., Bouchet-Marquis, C., Al-Amoudi, A. \& Livolant, F. (2007). How to 
'read' a vitreous section. Methods in Cell Biology 79, 385-406.

Erickson, H. P. (1997). FtsZ, a tubulin homologue in prokaryote cell division. Trends in Cell Biology 7, 362-367.

Fan, G. Y., Mercurio, P. J., Young, S. J. \& Ellisman, M. H. (1993). Telemicroscopy. Ultramicroscopy 52, 499-503.

Fernandez, J. J., Li, S. \& Crowther, R. A. (2006). CTF determination and correction in electron cryotomography. Ultramicroscopy 106, 587-596.

Figge, R. M., Divakaruni, A. V. \& Gober, J. W. (2004). $\mathrm{MreB}$, the cell shape-determining bacterial actin homologue, co-ordinates cell wall morphogenesis in Caulobacter crescentus. Molecular Microbiology 51, 1321-1332.

Forster, F. (2005). Quantitative Analysis of Macromolecules in Cryoelectron Tomograms using Correlation Methods. Technical University of Munich, Munich, Germany.

Frangakis, A. S. \& Hegerl, R. (2001). Noise reduction in electron tomographic reconstructions using nonlinear anisotropic diffusion. Journal of Structural Biology 135, 239-250.

Frank, J. (2006). Electron Tomography: Methods for ThreeDimensional Visualization of Structures in the Cell, 2nd edn. London, New York: Springer.

Frederik, P. M. \& Hubert, D. H. (2005). Cryoelectron microscopy of liposomes. Methods in Ensymology 391, 431-448.

Fu, C. Y., Wang, K., Gan, L., Lanman, J., Khayat, R., Young, M. J., Jensen, G. J., Doerschuk, P. C. \& JoHnson, J. E. (2010). In vivo assembly of an archaeal virus studied with whole-cell electron cryotomography. Structure 18, 1579-1586.

Gaietta, G., Deerinck, T. J., Adams, S. R., Bouwer, J., Tour, O., Laird, D. W., Sosinsky, G. E., Tsien, R. Y. \& Ellisman, M. H. (2002). Multicolor and electron microscopic imaging of connexin trafficking. Science 296, 503-507.

Gan, L., Ladinsky, M. S. \& Jensen, G. J. (2011). Organization of the smallest eukaryotic spindle. Current Biology 21, 1578-1583.

Garner, E. C., Bernard, R., Wang, W., Zhuang, X., Rudner, D. Z. \& Mitchison, T. (2011). Coupled, circumferential motions of the cell wall synthesis machinery and MreB filaments in B. subtilis. Science 333, 222-225.

Gilbert, P. (1972). Iterative methods for the threedimensional reconstruction of an object from projections. Journal of Theoretical Biology 36, 105-117.

Gilkey, J. C. \& Staehelin, L. A. (1986). Advances in ultrarapid freezing for the preservation of cellular ultrastructure. Journal of Electron Microscopy Technique 3, $177-210$.

GitAi, Z. (2005). The new bacterial cell biology: moving parts and subcellular architecture. Cell 120, 577-586.

Goodsell, D. S. (2005). Visual methods from atoms to cells. Structure 13, 347-354.
Grunewald, K., Desai, P., Winkler, D. C., Heymann, J. B., Belnap, D. M., Baumeister, W. \& Steven, A. C. (2003). Three-dimensional structure of herpes simplex virus from cryo-electron tomography. Science 302, 1396-1398.

Gruska, M., Medalia, O., Baumeister, W. \& Leis, A. (2008). Electron tomography of vitreous sections from cultured mammalian cells. Journal of Structural Biology 161, 384-392.

Harris, A., Cardone, G., Winkler, D. C., Heymann, J. B., Brecher, M., White, J. M. \& Steven, A. C. (2006). Influenza virus pleiomorphy characterized by cryoelectron tomography. Proceedings of the National Academy of Sciences of the United States of America 103, 19123-19127.

Hayles, M. F., Matthijs De Winter, D. A., Schnejdenberg, C. T., Meeldijk, J. D., Luecken, U., Persoon, H., De Water, J., De Jong, F., Humbel, B. M. \& VerkleiJ, A. J. (2010). The making of frozenhydrated, vitreous lamellas from cells for cryo-electron microscopy. Journal of Structural Biology 172, 180-190.

He, W., Cowin, P. \& Stokes, D. L. (2003). Untangling desmosomal knots with electron tomography. Science 302, 109-113.

He, W., Kivork, C., Machinani, S., Morphew, M. K., Gail, A. M., Tesar, D. B., Tiangco, N. E., Mcintosh, J. R. \& Bjorkman, P. J. (2007). A freeze substitution fixation-based gold enlarging technique for EM studies of endocytosed nanogold-labeled molecules. Journal of Structural Biology 160, 103-113.

He, W., Ladinsky, M. S., Huey-Tubman, K. E., Jensen, G. J., Mcintosh, J. R. \& Bjorkman, P. J. (2008). FcRnmediated antibody transport across epithelial cells revealed by electron tomography. Nature 455, 542-546.

Henderson, G. P., Gan, L. \& Jensen, G. J. (2007). 3-D ultrastructure of $O$. tauri: electron cryotomography of an entire eukaryotic cell. PloS ONE 2, e749.

Henderson, R., Baldwin, J. M., Ceska, T. A., Zemlin, F., Beckmann, E. \& Downing, K. H. (1990). Model for the structure of bacteriorhodopsin based on high-resolution electron cryo-microscopy. Journal of Molecular Biology 213, 899-929.

Herman, G. T., Lent, A. \& Rowland, S. W. (1973). ART: mathematics and applications. A report on the mathematical foundations and on the applicability to real data of the algebraic reconstruction techniques. Journal of Theoretical Biology 42, 1-32.

Heymann, J. B., Cardone, G., Winkler, D. C. \& Steven, A. C. (2008). Computational resources for cryo-electron tomography in Bsoft. Journal of Structural Biology 161, 232-242.

Hoppe, W., Gassmann, J., Hunsmann, N., Schramm, H. J. \& STURM, M. (1974). Three-dimensional reconstruction of individual negatively stained yeast fatty-acid synthetase molecules from tilt series in the electron microscope. Hoppe-Seylers Zeitschrift für Physiologische Chemie 355, 1483-1487. 
Hitien, C. E., Marko, M., Frank, J. \& Mannella, C. A. (2002). Electron tomographic analysis of frozenhydrated tissue sections. Journal of Structural Biology 138, 63-73.

Iancu, C. V., Morris, D. M., Dou, Z., Heinhorst, S., Cannon, G. C. \& Jensen, G. J. (2010). Organization, structure, and assembly of alpha-carboxysomes determined by electron cryotomography of intact cells. Journal of Molecular Biology 396, 105-117.

Iancu, C. V., Tivol, W. F., Schooler, J. B., Dias, D. P., Henderson, G. P., Murphy, G. E., Wright, E. R., Li, Z., Yu, Z., Briegel, A., Gan, L., He, Y. \& Jensen, G. J. (2006a). Electron cryotomography sample preparation using the Vitrobot. Nature Protocols 1, 2813-2819.

Iancu, C. V., Wright, E. R., Benjamin, J., Tivol, W. F., Dias, D. P., Murphy, G. E., Morrison, R. C., Heymann, J. B. \& Jensen, G. J. (2005). A 'flip-flop' rotation stage for routine dual-axis electron cryotomography. Journal of Structural Biology 151, 288-297.

Iancu, C. V., Wright, E. R., Heymann, J. B. \& Jensen, G. J. (2006b). A comparison of liquid nitrogen and liquid helium as cryogens for electron cryotomography. Journal of Structural Biology 153, 231-240.

Ingerson-Mahar, M., Briegel, A., Werner, J. N., Jensen, G. J. \& Gitai, Z. (2010). The metabolic enzyme CTP synthase forms cytoskeletal filaments. Nature Cell Biology 12, 739-746.

Jones, L. J., Carballido-Lopez, R. \& Errington, J. (2001). Control of cell shape in bacteria: helical, actinlike filaments in Bacillus subtilis. Cell 104, 913-922.

Khayat, R., Tang, L., Larson, E. T., Lawrence, C. M., Young, M. \& Johnson, J. E. (2005). Structure of an archaeal virus capsid protein reveals a common ancestry to eukaryotic and bacterial viruses. Proceedings of the National Academy of Sciences of the United States of America 102, 18944-18949.

Khursigara, C. M., Wu, X. \& Subramaniam, S. (2008a). Chemoreceptors in Caulobacter crescentus: trimers of receptor dimers in a partially ordered hexagonally packed array. Journal of Bacteriology 190, 6805-6810.

Khursigara, C. M., Wu, X., Zhang, P., Lefman, J. \& Subramaniam, S. (2008b). Role of HAMP domains in chemotaxis signaling by bacterial chemoreceptors. Proceedings of the National Academy of Sciences, USA 105, 16555-16560.

Komeili, A., Li, Z., Newman, D. K. \& Jensen, G. J. (2006). Magnetosomes are cell membrane invaginations organized by the actin-like protein MamK. Science 311, 242-245.

Koster, A. J., Grimm, R., Typke, D., Hegerl, R., Stoschek, A., Walz, J. \& Baumeister, W. (1997). Perspectives of molecular and cellular electron tomography. Journal of Structural Biology 120, 276-308.

Kremer, J. R., Mastronarde, D. N. \& Mcintosh, J. R. (1996). Computer visualization of three-dimensional image data using IMOD. Journal of Structural Biology 116, $71-76$.

Kudryashev, M., CyrklafF, M., Wallich, R., Baumeister, W. \& FrischKnecht, F. (2010). Distinct in situ structures of the Borrelia flagellar motor. Journal of Structural Biology 169, 54-61.

Kurner, J., Frangakis, A. S. \& Baumeister, W. (2005). Cryo-electron tomography reveals the cytoskeletal structure of Spiroplasma melliferum. Science 307, 436-438.

Ladinsky, M. S. \& Howell, K. E. (2007). Electron tomography of immunolabeled cryosections. Methods in Cell Biology 79, 543-558.

Ladinsky, M. S., Mastronarde, D. N., Mcintosh, J. R., Howell, K. E. \& Staehelin, L. A. (1999). Golgi structure in three dimensions: functional insights from the normal rat kidney cell. Journal of Cell Biology 144, 1135-1149.

Ladinsky, M. S., Pierson, J. M. \& Mcintosh, J. R. (2006). Vitreous cryo-sectioning of cells facilitated by a micromanipulator. Journal of Microscopy 224, 129-134.

Lawrence, A., Bouwer, J. C., Perkins, G. \& Ellisman, M. H. (2006). Transform-based backprojection for volume reconstruction of large format electron microscope tilt series. Journal of Structural Biology 154, 144-167.

Lee, E., Fahimian, B. P., Iancu, C. V., Suloway, C., Murphy, G. E., Wright, E. R., Castano-Diez, D., Jensen, G. J. \& Miao, J. (2008). Radiation dose reduction and image enhancement in biological imaging through equally-sloped tomography. Journal of Structural Biology 164, 221-227.

Lenzi, D., Crum, J., Ellisman, M. H. \& Roberts, W. M. (2002). Depolarization redistributes synaptic membrane and creates a gradient of vesicles on the synaptic body at a ribbon synapse. Neuron 36, 649-659.

Lenzi, D., Runyeon, J. W., Crum, J., Ellisman, M. H. \& RoberTs, W. M. (1999). Synaptic vesicle populations in saccular hair cells reconstructed by electron tomography. Journal of Neuroscience 19, 119-132.

Li, Z., Trimble, M. J., Brun, Y. V. \& Jensen, G. J. (2007). The structure of FtsZ filaments in vivo suggests a forcegenerating role in cell division. EMBO Journal 26(22), 4694-4708.

Liu, H., Jin, L., Koh, S. B., Atanasov, I., Schein, S., Wu, L. \& ZHou, Z. H. (2010). Atomic structure of human adenovirus by cryo-EM reveals interactions among protein networks. Science 329, 1038-1043.

Liu, J., Lin, T., Botkin, D. J., Mccrum, E., Winkler, H. \& Norris, S. J. (2009). Intact flagellar motor of Borrelia burgdorferi revealed by cryo-electron tomography: evidence for stator ring curvature and rotor/C-ring assembly flexion. Journal of Bacteriology 191, 5026-5036.

Lucic, V., Forster, F. \& Baumeister, W. (2005). Structural studies by electron tomography: from cells to molecules. Annual Review of Biochemistry 74, 833-865.

Majorovits, E., Barton, B., Schultheiss, K., PerezWillard, F., Gerthsen, D. \& Schroder, R. R. (2007). 
Optimizing phase contrast in transmission electron microscopy with an electrostatic (Boersch) phase plate. Ultramicroscopy 107, 213-226.

Mandelkow, E. M., Mandelkow, E. \& Milligan, R. A. (1991). Microtubule dynamics and microtubule caps: a time-resolved cryo-electron microscopy study. Journal of Cell Biology 114, 977-991.

Marko, M., Hsieh, C., Schalek, R., Frank, J. \& Mannella, C. (2007). Focused-ion-beam thinning of frozen-hydrated biological specimens for cryo-electron microscopy. Nature Methods 4, 215-217.

Marsh, B. J. (2007). Reconstructing mammalian membrane architecture by large area cellular tomography. Methods in Cell Biology 79, 193-220.

Marsh, B. J., Mastronarde, D. N., Buttle, K. F., Howell, K. E. \& Mcintosh, J. R. (2001). Organellar relationships in the Golgi region of the pancreatic beta cell line, HIT-T15, visualized by high resolution electron tomography. Proceedings of the National Academy of Sciences of the United States of America 98, 2399-2406.

Marsh, B. J., Volkmann, N., Mcintosh, J. R. \& Howell, K. E. (2004). Direct continuities between cisternae at different levels of the Golgi complex in glucosestimulated mouse islet beta cells. Proceedings of the National Academy of Sciences of the United States of America 101, 5565-5570.

Masich, S., Ostberg, T., Norlen, L., Shupliakov, O. \& Daneholt, B. (2006). A procedure to deposit fiducial markers on vitreous cryo-sections for cellular tomography. Journal of Structural Biology 156(3), 461-468.

Mastronarde, D. N. (1997). Dual-axis tomography: an approach with alignment methods that preserve resolution. Journal of Structural Biology 120, 343-352.

Mastronarde, D. N. (2005). Automated electron microscope tomography using robust prediction of specimen movements. Journal of Structural Biology 152, 36-51.

Mastronarde, D. N., Ladinsky, M. S. \& Mcintosh, J. R. (2000). Super-thin serial sectioning for high resolution 3-D reconstruction of cellular structures. Microscopy and Microanalysis, 3, 221-222.

Mcdonald, K. L. (2009). A review of high-pressure freezing preparation techniques for correlative light and electron microscopy of the same cells and tissues. Journal of microscopy 235, 273-281.

Mcdonald, K. L. \& Auer, M. (2006). High-pressure freezing, cellular tomography, and structural cell biology. Biotechniques 41, 137-143.

Mcewen, B. F., Downing, K. H. \& Glaeser, R. M. (1995). The relevance of dose-fractionation in tomography of radiation-sensitive specimens. Ultramicroscopy $\mathbf{6 0}$, 357-373.

Mcewen, B. F., Radermacher, M., Rieder, C. L. \& Frank, J. (1986). Tomographic three-dimensional reconstruction of cilia ultrastructure from thick sections. Proceedings of the National Academy of Sciences of the United States of America 83, 9040-9044.
Mcintosh, J. R., Grishchuk, E. L., Morphew, M. K., Efremov, A. K., Zhudenkov, K., Volkov, V. A., Cheeseman, I. M., Desai, A., Mastronarde, D. N. \& Ataullakhanov, F. I. (2008). Fibrils connect microtubule tips with kinetochores: a mechanism to couple tubulin dynamics to chromosome motion. Cell 135 , 322-333.

Mcintosh, J. R., Roos, U. P., Neighbors, B. \& Mcdonald, K. L. (1985). Architecture of the microtubule component of mitotic spindles from Dictyostelium discoideum. Journal of Cell Science 75, 93-129.

Mcmullan, G., Cattermole, D. M., Chen, S., Henderson, R., Llopart, X., Summerfield, C., Tlustos, L. \& FAruQI, A. R. (2007). Electron imaging with Medipix2 hybrid pixel detector. Ultramicroscopy 107, 401-413.

Mcmullan, G., Clark, A. T., Turchetta, R. \& Faruqi, A. R. (2009). Enhanced imaging in low dose electron microscopy using electron counting. Ultramicroscopy 109, 1411-1416.

Medalia, O., Weber, I., Frangakis, A. S., Nicastro, D., Gerisch, G. \& Baumeister, W. (2002). Macromolecular architecture in eukaryotic cells visualized by cryoelectron tomography. Science 298, 1209-1213.

Mercogliano, C. P. \& Derosier, D. J. (2007). Concatenated metallothionein as a clonable gold label for electron microscopy. Journal of Structural Biology 160, $70-82$.

Messaoudi, C., Boudier, T., Sanchez Sorzano, C. O. \& Marco, S. (2007). TomoJ: tomography software for three-dimensional reconstruction in transmission electron microscopy. BMC Bioinformatics 8, 288.

Milazzo, A. C., Leblanc, P., Duttweiler, F., Jin, L., Bouwer, J. C., Peltier, S., Ellisman, M., Bieser, F., Matis, H. S., Wieman, H., Denes, P., Kleinfelder, S. \& Xuong, N. H. (2005). Active pixel sensor array as a detector for electron microscopy. Ultramicroscopy 104, 152-159.

Milne, J. L. \& Subramaniam, S. (2009). Cryo-electron tomography of bacteria: progress, challenges and future prospects. Nature Reviews Microbiology 7, 666-675.

Minton, A. P. (2006). How can biochemical reactions within cells differ from those in test tubes? Journal of Cell Science 119, 2863-2869.

Moor, H. (1987). Theory and Practice of High Pressure Freezing. Berlin: Springer-Verlag.

Moritz, M., Braunfeld, M. B., Guenebaut, V., Heuser, J. \& Agard, D. A. (2000). Structure of the gamma-tubulin ring complex: a template for microtubule nucleation. Nature Cell Biology 2, 365-370.

Morphew, M., He, W., Bjorkman, P. J. \& Mcintosh, J. R. (2008). Silver enhancement of Nanogold particles during freeze substitution for electron microscopy. Journal of Microscopy 230, 263-267. 
Murphy, G. E. \& Jensen, G. J. (2005). Electron cryotomography of the E. coli pyruvate and 2-oxoglutarate dehydrogenase complexes. Structure 13, 1765-1773.

Murphy, G. E., Leadbetter, J. R. \& Jensen, G. J. (2006). In situ structure of the complete Treponema primitia flagellar motor. Nature 442, 1062-1064.

Murphy, G. E., Lowekamp, B. C., Zerfas, P. M., Chandler, R. J., Narasimha, R., Venditti, C. P. \& Subramaniam, S. (2010). Ion-abrasion scanning electron microscopy reveals distorted liver mitochondrial morphology in murine methylmalonic acidemia. Journal of Structural Biology 171, 125-132.

Murphy, G. E., Matson, E. G., Leadbetter, J. R., Berg, H. C. \& Jensen, G. J. (2008). Novel ultrastructures of Treponema primitia and their implications for motility. Molecular Microbiology 67, 1184-1195.

Nagayama, K. \& Danev, R. (2009). Phase-plate electron microscopy: a novel imaging tool to reveal close-to-life nano-structures. Biophysical Reviews 1, 37-42.

Nickell, S., Forster, F., Linaroudis, A., Net, W. D., Beck, F., Hegerl, R., Baumeister, W. \& Plitzko, J. M. (2005). TOM software toolbox: acquisition and analysis for electron tomography. Journal of Structural Biology 149(3), 227-234.

Nickell, S., Kofler, C., Leis, A. P. \& Baumeister, W. (2006). A visual approach to proteomics. Nature Reviews Molecular Cell Biology 7, 225-230.

Noske, A. B., Costin, A. J., Morgan, G. P. \& Marsh, B. J. (2008). Expedited approaches to whole cell electron tomography and organelle mark-up in situ in highpressure frozen pancreatic islets. Journal of Structural Biology 161, 298-313.

O’toole, E. T., Mcdonald, K. L., Mantler, J., Mcintosh, J. R., Hyman, A. A. \& Muller-Reichert, T. (2003). Morphologically distinct microtubule ends in the mitotic centrosome of Caenorbabditis elegans. Journal of Cell Biology 163, 451-456.

O'toole, E. T., Winey, M. \& Mcintosh, J. R. (1999). High-voltage electron tomography of spindle pole bodies and early mitotic spindles in the yeast Saccharomyces cerevisiae. Molecular Biology Cell 10, 2017-2031.

Olins, D. E., Olins, A. L., Levy, H. A., Durfee, R. C., Margle, S. M., Tinnel, E. P. \& Dover, S. D. (1983). Electron microscope tomography: transcription in three dimensions. Science 220, 498-500.

Ortiz, J. O., Brandt, F., Matias, V. R., Sennels, L., Rappsilber, J., Scheres, S. H., Eibauer, M., Hartl, F. U. \& Baumeister, W. (2010). Structure of hibernating ribosomes studied by cryoelectron tomography in vitro and in situ. Journal of Cell Biology 190, 613-621.

Ortiz, J. O., Forster, F., Kurner, J., Linaroudis, A. A. \& Baumeister, W. (2006). Mapping $70 \mathrm{~S}$ ribosomes in intact cells by cryoelectron tomography and pattern recognition. Journal of Structural Biology 156, 334-341.
Overby, A. K., Pettersson, R. F., Grunewald, K. \& Huiskonen, J. T. (2008). Insights into bunyavirus architecture from electron cryotomography of Uukuniemi virus. Proceedings of the National Academy of Sciences of the United States of America 105, 2375-2379.

Patla, I., Volberg, T., Elad, N., Hirschfeld-Warneken, V., Grashoff, C., Fassler, R., Spatz, J. P., Geiger, B. \& Medalia, O. (2010). Dissecting the molecular architecture of integrin adhesion sites by cryo-electron tomography. Nature Cell Biology 12, 909-915.

Penczek, P. A. (2010a). Fundamentals of threedimensional reconstruction from projections. Methods in Enzymology 482, 1-33.

Penczek, P. A. (2010b). Image restoration in cryo-electron microscopy. Methods in Enaymology 482, 35-72.

Pettersen, E. F., Goddard, T. D., Huang, C. C., Couch, G. S., Greenblatt, D. M., Meng, E. C. \& Ferrin, T. E. (2004). UCSF Chimera - a visualization system for exploratory research and analysis. Journal of Computational Chemistry 25, 1605-1612.

Pierson, J., Fernandez, J. J., Bos, E., Amini, S., Gnaegi, H., Vos, M., Bel, B., Adolfsen, F., Carrascosa, J. L. \& Peters, P. J. (2010). Improving the technique of vitreous cryo-sectioning for cryo-electron tomography: electrostatic charging for section attachment and implementation of an anti-contamination glove box. Journal of Structural Biology 169, 219-225.

Pilhofer, M., Ladinsky, M. S., Mcdowall, A. W. \& Jensen, G. J. (2010). Bacterial TEM: new insights from cryo-microscopy. Methods in Cell Biology 96, 21-45.

Ress, D., Harlow, M. L., Schwarz, M., Marshall, R. M. \& Mcmahan, U. J. (1999). Automatic acquisition of fiducial markers and alignment of images in tilt series for electron tomography. Journal of Electron Microscopy (Tokyo) 48, 277-287.

Rigort, A., Bauerlein, F. J., Leis, A., Gruska, M., Hoffmann, C., Laugks, T., Bohm, U., Eibauer, M., Gnaegi, H., Baumeister, W. \& Plitzko, J. M. (2010). Micromachining tools and correlative approaches for cellular cryo-electron tomography. Journal of Structural Biology 172, 169-179.

Salje, J., Zuber, B. \& Lowe, J. (2009). Electron cryomicroscopy of E. coli reveals filament bundles involved in plasmid DNA segregation. Science 323, 509-512.

Sandberg, K. \& Brega, M. (2007). Segmentation of thin structures in electron micrographs using orientation fields. Journal of Structural Biology 157, 403-415.

Saxton, W. O., Baumeister, W. \& Hahn, M. (1984). Three-dimensional reconstruction of imperfect twodimensional crystals. Ultramicroscopy 13, 57-70.

Scheffel, A., Gruska, M., Faivre, D., Linaroudis, A., Plitzko, J. M. \& Schuler, D. (2006). An acidic protein aligns magnetosomes along a filamentous structure in magnetotactic bacteria. Nature 440, 110-114.

Scheres, S. H., Melero, R., Valle, M. \& Carazo, J. M. (2009). Averaging of electron subtomograms and 
random conical tilt reconstructions through likelihood optimization. Structure 17, 1563-1572.

Schwartz, C. L., Sarbash, V. I., Ataullakhanov, F. I., Mcintosh, J. R. \& Nicastro, D. (2007). Cryofluorescence microscopy facilitates correlations between light and cryo-electron microscopy and reduces the rate of photobleaching. Journal of Microscopy 227, 98-109.

Seybert, A., Herrmann, R. \& Frangakis, A. S. (2006). Structural analysis of Mycoplasma pneumoniae by cryo-electron tomography. Journal of Structural Biology 156, 342-354.

Shaikh, T. R., Gao, H., Baxter, W. T., Asturias, F. J., Boisset, N., Leith, A. \& Frank, J. (2008). SPIDER image processing for single-particle reconstruction of biological macromolecules from electron micrographs. Nature Protocol 3, 1941-1974.

Shin, Y. L., Le, T. \& Rothfield, L. (2003). Division site selection in Escherichia coli involves dynamic redistribution of Min proteins within coiled structures that extend between the two cell poles. Proceedings of the National Academy of Sciences, USA 100(13), 7865-7870.

Sigworth, F. J. (1998). A maximum-likelihood approach to single-particle image refinement. Journal of Structural Biology 122, 328-339.

Skoglund, U., Andersson, K., Strandberg, B. \& Daneholt, B. (1986). Three-dimensional structure of a specific pre-messenger RNP particle established by electron microscope tomography. Nature 319, 560-564.

Soto, G. E., Young, S. J., Martone, M. E., Deerinck, T. J., Lamont, S., Carragher, B. O., Hama, K. \& Ellisman, M. H. (1994). Serial section electron tomography: a method for three-dimensional reconstruction of large structures. Neuroimage 1, 230-243.

Sougrat, R., Bartesaghi, A., Lifson, J. D., Bennett, A. E., Bess, J. W., Zabransky, D. J. \& Subramaniam, S. (2007). Electron tomography of the contact between T cells and SIV/HIV-1: implications for viral entry. PLoS Pathogen 3, e63.

Srivastava, P., Demarre, G., Karpova, T. S., Mcnally, J. \& Chattoraj, D. K. (2007). Changes in nucleoid morphology and origin localization upon inhibition or alteration of the actin homolog, MreB, of Vibrio cholerae. Journal of Bacteriology 189, 7450-7463.

Suloway, C., Shi, J., Cheng, A., Pulokas, J., Carragher, B., Potter, C.S., Zheng, S. Q., Agard, D. A. \& Jensen, G. J. (2009). Fully automated, sequential tiltseries acquisition with Leginon. Journal of Structural Biology 167, 11-18.

Swulius, M. T., Chen, S., Jane Ding, H., Li, Z., Briegel, A., Pilhofer, M., Tocheva, E. I., Lybarger, S. R., Johnson, T. L., SAndkvist, M. \& Jensen, G. J. (2011). Long helical filaments are not seen encircling cells in electron cryotomograms of rod-shaped bacteria. Biochemical and Biophysical Research Communications 407, 650-655.
TAng, H., Braun, T. F. \& Blair, D. F. (1996). Motility protein complexes in the bacterial flagellar motor. Journal of Molecular Biology 261, 209-221.

TAYlor, K. A. \& Glaeser, R. M. (1974). Electron diffraction of frozen, hydrated protein crystals. Science 186, 1036-1037.

Thomas, D., Morgan, D. G. \& Derosier, D. J. (2001). Structures of bacterial flagellar motors from two FliF-FliG gene fusion mutants. Journal of Bacteriology 183, 6404-6412.

Tocheva, E. I., Li, Z. \& Jensen, G. J. (2010). Electron cryotomography. Cold Spring Harbor Perspectives in Biology 2, a003442.

Tokuyasu, K. T. (1986). Application of cryoultramicrotomy to immunocytochemistry. Journal of Microscopy 143, 139-149.

Trucco, A., Polishchuk, R. S., Martella, O., Di Pentima, A., Fusella, A., Di Giandomenico, D., San Pietro, E., Beznoussenko, G. V., Polishchuk, E. V., Baldassarre, M., Buccione, R., Geerts, W. J., Koster, A. J., Burger, K. N., Mironov, A. A. \& Luini, A. (2004). Secretory traffic triggers the formation of tubular continuities across Golgi sub-compartments. Nature Cell Biology 6, 1071-1081.

Urban, E., Jacob, S., Nemethova, M., Resch, G. P. \& SmaLL, J. V. (2010). Electron tomography reveals unbranched networks of actin filaments in lamellipodia. Nature Cell Biology 12, 429-435.

Van Der Heide, P., Xu, X. P., Marsh, B. J., Hanein, D. \& Volkmann, N. (2007). Efficient automatic noise reduction of electron tomographic reconstructions based on iterative median filtering. Journal of Structural Biology 158, 196-204.

Vandenbeldt, K. J., Barnard, R. M., Hergert, P. J., Meng, X., Maiato, H. \& Mcewen, B. F. (2006). Kinetochores use a novel mechanism for coordinating the dynamics of individual microtubules. Current Biology 16, 1217-1223.

Volkmann, N. (2002). A novel three-dimensional variant of the watershed transform for segmentation of electron density maps. Journal of Structural Biology 138, 123-129.

Wang, Q., Mercogliano, C. P. \& Lowe, J. (2011). A ferritin-based label for cellular electron cryotomography. Structure 19, 147-154.

Wei, D. Y. \& Yin, C. C. (2010). An optimized locally adaptive non-local means denoising filter for cryoelectron microscopy data. Journal of Structural Biology 172, 211-218.

White, J. G., Southgate, E., Thomson, J. N. \& Brenner, S. (1986). The structure of the nervous system of the nematode Caenorbabditis elegans. Philosophical Transactions of the Royal Society of London. B, Biological Sciences 314, $1-340$.

White, T. A., Bartesaghi, A., Borgnia, M. J., Meyerson, J. R., De La Cruz, M. J., Bess, J. W., Nandwani, R., 
Hoxie, J. A., Lifson, J. D., Milne, J. L. \& Subramaniam, S. (2010). Molecular architectures of trimeric SIV and HIV-1 envelope glycoproteins on intact viruses: strain-dependent variation in quaternary structure. PLoS Pathogens 6, e1001249.

Winey, M., Mamay, C. L., O’toole, E. T., Mastronarde, D. N., Giddings, T. H., JR., Mcdonald, K. L. \& Mcintosh, J. R. (1995). Three-dimensional ultrastructural analysis of the Saccharomyces cerevisiae mitotic spindle. Journal of Cell Biology 129, 1601-1615.

WinKLER, H. (2007). 3D reconstruction and processing of volumetric data in cryo-electron tomography. Journal of Structural Biology 157, 126-137.

WinkLer, H. \& TAYLOR, K. A. (2006). Accurate markerfree alignment with simultaneous geometry determination and reconstruction of tilt series in electron tomography. Ultramicroscopy 106, 240-254.

Wolf, M., Garcea, R. L., GrigoriefF, N. \& Harrison, S. C. (2010). Subunit interactions in bovine papillomavirus. Proceedings of the National Academy of Sciences of the United States of America 107, 6298-6303.

Wright, E. R., Schooler, J. B., Ding, H. J., Kieffer, C., Fillmore, C., Sundquist, W. I. \& Jensen, G. J. (2007). Electron cryotomography of immature HIV-1 virions reveals the structure of the CA and SP1 Gag shells. EMBO J 26, 2218-2226.
Xiong, Q., Morphew, M. K., Schwartz, C. L., Hoenger, A. H. \& Mastronarde, D. N. (2009). CTF determination and correction for low dose tomographic tilt series. Journal of Structural Biology 168, 378-387.

Yang, Q., Rout, M. P. \& Akey, C. W. (1998). Threedimensional architecture of the isolated yeast nuclear pore complex: functional and evolutionary implications. Molecular Cell 1, 223-234.

Zanetti, G., Riches, J. D., Fuller, S. D. \& Briggs, J. A. (2009). Contrast transfer function correction applied to cryo-electron tomography and sub-tomogram averaging. Journal of Structural Biology 168, 305-312.

Zhang, P., Bos, E., Heymann, J., Gnaegi, H., Kessel, M., Peters, P. J. \& Subramaniam, S. (2004). Direct visualization of receptor arrays in frozen-hydrated sections and plunge-frozen specimens of $E$. coli engineered to overproduce the chemotaxis receptor Tsr. Journal of Microscopy 216, 76-83.

Zheng, Q. S., Braunfeld, M. B., Sedat, J. W. \& Agard, D. A. (2004). An improved strategy for automated electron microscopic tomography. Journal of Structural Biology 147, 91-101.

Zheng, S. Q., Matsuda, A., Braunfeld, M. B., Sedat, J. W. \& Agard, D. A. (2009). Dual-axis target mapping and automated sequential acquisition of dual-axis EM tomographic data. Journal of Structural Biology 168, 323-331. 